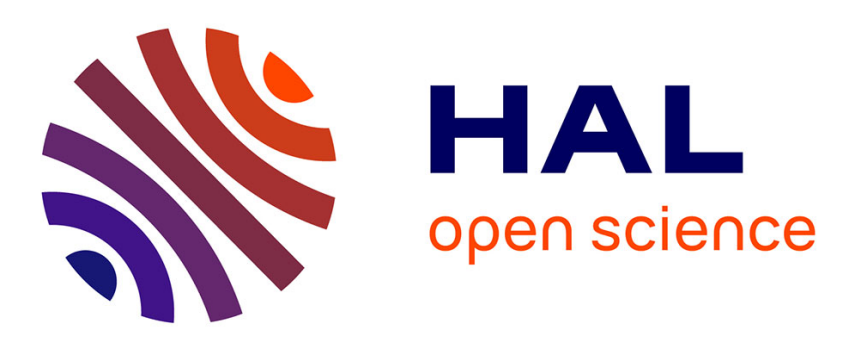

\title{
Numerical investigations of separation-induced transition on high-lift low-pressure turbine using RANS and LES methods
}

J Marty

\section{- To cite this version:}

J Marty. Numerical investigations of separation-induced transition on high-lift low-pressure turbine using RANS and LES methods. Proceedings of the Institution of Mechanical Engineers, Part A: Journal of Power and Energy, 2014, 39 p. 10.1177/0957650914548741 . hal-01080267

\section{HAL Id: hal-01080267 \\ https://hal.science/hal-01080267}

Submitted on 4 Nov 2014

HAL is a multi-disciplinary open access archive for the deposit and dissemination of scientific research documents, whether they are published or not. The documents may come from teaching and research institutions in France or abroad, or from public or private research centers.
L'archive ouverte pluridisciplinaire HAL, est destinée au dépôt et à la diffusion de documents scientifiques de niveau recherche, publiés ou non, émanant des établissements d'enseignement et de recherche français ou étrangers, des laboratoires publics ou privés. 


\title{
Numerical Investigations of Separation-Induced Transition on High-Lift Low-Pressure Turbine using RANS and LES methods
}

\author{
J. Marty, \\ ONERA - The French Aerospace Lab, \\ F-92190 Meudon, France, e-mail: Julien.Marty@onera.fr
}

\begin{abstract}
At low Reynolds numbers, laminar-turbulent transition occurs on the suction side of high-lift low-pressure turbine blades. The prediction of this flow is an important step in low-pressure turbine design. Thus the laminar-turbulent transition must be modeled or resolved. The flow around the high-lift low-pressure turbine blade T106C is predicted using RANS simulations, without and with transition model, and Large-Eddy Simulations (LES). Large-eddy simulations are performed in order to predict the laminar separation bubble without any laminar-turbulent transition modeling. Only two Reynolds numbers are investigated with LES and the current study concerns also the validation of the turbulent random flow generation technique of Smirnov et al. [1]. Reynolds number and freestream turbulence effects are studied using the analysis of the unsteady behavior of the separated shear layer and the bubble. The steady flow predicted by RANS simulation with transition model and by the time-averaged LES are in good agreement with isentropic Mach number distribution at midspan, except for the lowest Reynolds number $\left(\operatorname{Re}_{2 i s}=80000\right)$. For this last case, the separation and transition points are predicted downstream of the experimental points. The spectral analysis of LES results at different locations allows determining specific frequencies of physical mechanisms. Large-eddy simulations are able to predict laminar separation bubble over the high-lift low-pressure turbine blade T106C as RANS simulation with transition model and to capture the Kelvin-Helmholtz instability which is the cause of the transition mechanism.
\end{abstract}

\section{Keywords}

Low pressure turbine, High lift airfoil, T106C turbine, Large-Eddy Simulation, KelvinHelmholtz instability, Spectral analysis, Transition modeling, Separation-induced transition

\section{Introduction}

In order to decrease the weight and cost of modern low-pressure turbines (LPT), the trend is to design LPT with a reduced number of blades while maintaining the same amount of stage work and a high efficiency. The trend of a drastic reduction of blade number is now over because a very small blade number has a too strong impact on performance, especially on efficiency. The engine manufacturers design now LPT with a decreased blade number with respect to the previous generation i.e. to the engine of the last decade but this blade number results from a compromise between weight and cost decrease, and performance increase as the objective of blade number reduction must be achieved with a maximum efficiency. So, by comparison to previous LPT, the blade loading and efficiency are higher while the weight and cost are lower. The modern LPT produces high lift and the suction side of the blade is subjected to strong adverse pressure gradient.

The LPT operates at relative low Reynolds number. At cruise, this Reynolds number is nearly 100000 [2]. The boundary layer is consequently laminar over a large part of the suction side and is unable to overcome the strong adverse pressure gradient. Thus the boundary layer may separate and create a laminar separation bubble (LSB) where the laminar-turbulent transition 
is triggered [3]. Depending on the Reynolds number, the adverse pressure gradient and the freestream turbulence, this bubble can be short, long or open. Gaster [4] conducted a detailed investigation of laminar separation bubble, showed the existence of these different bubbles and described the bursting phenomenon i.e. the passage from a short bubble to a long bubble. $\mathrm{He}$ also tried to identify critical parameters for the bursting onset and showed that for the longer bubbles and low freestream turbulence, much of the flow within the bubble is laminar and Tollmien-Schlichting instabilities have been observed. The phenomenon of transition triggered by separation bubble was also described by Hatman and Wang [5][6][7][8]. For constant freestream conditions (turbulence intensity and adverse pressure gradient) when lowering the Reynolds number the bubble size increases. Firstly, the bursting is observed, which means the change of a short bubble into a long bubble. Then, for extreme low Reynolds numbers massive separation takes place and the boundary layer does not reattach: the bubble is open. The lower the Reynolds number is, the higher are the losses and lower the efficiency [3]. It is worth noting that in the present study, the bubble classification is based on the effect of the bubble on the static pressure distribution. A short bubble causes a minor perturbation to the pressure distribution and the suction peak is retained and can increase with an increasing angle of attack. On the other hand, a long bubble drastically modifies the pressure distribution in such a way that the suction peak disappears [4].

Boundary layer prediction is a critical point for the flow simulation within a high lift LPT. The most accurate method is the Direct Numerical Simulation. Although some recent studies deal with turbomachine simulation using DNS and show the capability to correctly capture the laminar-turbulent transition phenomenon [9][10][11], the required computational effort is still far beyond the capabilities of modern supercomputers. A cheaper way to simulate the flow within a compressor or a turbine is Reynolds Averaged Navier-Stokes simulation (RANS). It requires transition modeling in order to take the laminar part of the boundary layer into account. This modeling is performed using transition criterion [12] or transport equations [13][14][15]. Nevertheless RANS simulations provide only information about the mean flow and turbulence is modeled. A solution between these two methods is Large-Eddy Simulation in which the large scales are resolved and only the effect of the small unresolved (subgrid) scales is modeled. This method does not require transition modeling for the case of separation-induced transition. Recent studies demonstrated the benefits of LES on complex flow prediction in turbomachinery applications [16][17][18] and in helicopter framework where the laminar separation bubble exists, especially near the leading edge in dynamic stall conditions. Richez et al. [19] showed that the transition of the suction-side boundary layer via a LSB near the leading edge can be captured by LES as proved by the very good agreement with a linear inviscid instability theory.

Laminar-turbulent transition was already investigated by some authors with LES. Raverdy et al. [20] studied the low-pressure turbine T106A at $\mathrm{R}_{2 \mathrm{is}}=160000$ and $\mathrm{M}_{\infty}=0.1$. The predicted laminar separation bubble is short and the separated shear layer rolls up and coherent structures are ejected. The authors showed that this roll-up is tied to the Kelvin-Helmholtz instability. They also performed a spectral analysis. The two observed frequencies are respectively relative to the flapping of the separated shear layer and to the vortex shedding at the trailing edge. The comparison of spectra shows a coupling between the bubble delimited by separation and reattachment points and the vortex shedding. Mittal et al. [21] studied also a low-pressure turbine blade and highlighted the mechanism from the separated shear layer to the three-dimensional vortex shedding via the laminar separation bubble at low Reynolds number and the Kelvin-Helmholtz instability at high Reynolds number. At low Reynolds number, the vortex shedding frequencies are determined by the spectral analysis which also shows that the flow over the suction surface is not turbulent. Matsuura and Kato [22] performed a LES over the low pressure turbine cascade T106 without and with freestream turbulence. Without turbulence, pressure waves propagate from the trailing edge to the 
leading edge and induce oscillation of separation bubble. These waves do not exist with freestream turbulence $(5 \%)$. They show that the coherent structures within the wake are clearly dependent on the freestream turbulence: two-dimensional without turbulence, fully three-dimensional with turbulence. Funazaki et al. [23] studied experimentally and numerically the effect of the freestream turbulence on the boundary layer development over the suction side of a low pressure turbine blade for several high lift conditions. The inlet turbulence is generated using the stochastic noise generation and radiation method [24]. The higher the freestream turbulence intensity is, the thinner and shorter is the laminar separation bubble. The shear layer and the laminar separation bubble are energized just downstream of the separation point. With high freestream turbulence intensity, the location of the maximum thickness of the separation bubble moves upstream, implying earlier transition. The spectral analysis exhibits the Kelvin-Helmholtz instability which is confirmed by criterion computations. The unsteady analysis shows the involved mechanism from roll-up of the shear layer to the vortex shedding. This mechanism becomes more complex with freestream turbulence. Other authors studied the influence of passing wakes on the boundary layer development on the T106 blade for different exit Reynolds numbers (Michelassi et al. [25]: $\operatorname{Re}_{2 \text { is }}=270$ 000, Sarkar and Voke [26]: $\operatorname{Re}_{2 \text { is }}=160$ 000). Michelassi et al. [25] show that a LES can predict a transition point with $0.1 \mathrm{C}_{\mathrm{ax}}$ delay in comparison to DNS due to filtered frequencies. They consider that a better resolution is necessary in the transitional portion of the suction side in order to capture these frequencies. High freestream turbulence intensity and incoming wakes were also investigated by Zhang and Hodson [27]. The unsteady transition mechanism is strongly modified in comparison to low freestream turbulence intensity. The time-averaged separation on suction surface is much smaller and the transition onset moves upstream.

In turbomachinery flows, the freestream turbulence intensity range is wide, ranging from some tenths per cent to ten or twenty per cent. Due to the receptivity of the boundary layer, this intensity plays a significant part in the development of the boundary layer and in the transition mode. As discussed by Mayle [3], three modes are observed in turbomachinery flows: natural transition (Tollmien-Schlichting wave), by-pass transition and separated-flow transition. The natural transition occurs at low freestream turbulence intensity while by-pass transition is triggered at high turbulence level. For the third case, the turbulence influences the separation and transition points and so the bubble type. Thus it is important to inject the correct value of freestream turbulence intensity in numerical simulations. Some techniques exist to generate freestream turbulence for Large-Eddy Simulation, especially the cyclic channel [28], the stochastic noise generation and radiation method [24] used by Funazaki et al. [23], the synthetic eddy method (SEM) [29][30] or the random flow generation [1]. A review of these methods is given by Tabor and Baba-Ahmadi [31]. The method of Smirnov et $a l$. [1] is chosen for its respect to the continuity equation and its simplicity to use. One of the objectives of the current investigation is the validation of the method of Smirnov et al. [1] for turbomachinery applications. For the third transition mode, two instabilities can lead to transition, the Tollmien-Schlichting (TS) and Kelvin-Helmholtz (KH) instabilities which can be linked. Diwan and Ramesh [32] show the primary instability mechanism is inflectional and this inviscid instability can be seen as an extension of the TS instability. They consider that the $\mathrm{KH}$ instability is dominant only when the separated shear layer is far from the wall. McAuliffe and Yaras [33] show that both the TS and KH instabilities can play a role in transition process if they occur at the similar frequency (case 1 in the original article). In another case (case 2), the KH instability is dominant.

The objective of the current investigation is the assessment of compressible LES ability to predict separation-induced transition using elsA software which is not dedicated to LES method, by comparison to experimental data and numerical results obtained with RANS and 
URANS simulations based on transport equations for transition modeling [13][14]. The open bubble is considered as a difficult simulation for compressible LES over turbine airfoil. The low pressure turbine is the T106C one [34] and is described in the first part of this article. The innovative aspect of the present study is the high speed test case of which experimental data have been very recently published [34]. Then the numerical methods and turbulence and transition models are detailed. The results shown in the present article concern not only the performances and the average flow but the analysis of unsteady flow and spectral analysis for different Reynolds numbers and freestream intensities as well. This is worth noting that the present inlet conditions are not fully representative of real engine which involves high freestream turbulence intensity and incoming wakes. Nevertheless the aim of this investigation is to assess the ability of LES method in elsA CFD software to capture such flows, especially the open bubble which is only observed at low freestream turbulence intensity and low Reynolds number. The incoming wakes and high freestream turbulence intensity will be investigated in future works as the present investigation is only a part of a large internal project into laminar-turbulent transition in framework of high speed low pressure turbine characterized by high lift airfoil.

\section{T106C Cascade}

The T106C is classified as a very high-lift mid-loaded LP turbine airfoil, characterized by a suction side velocity peak at mid curvilinear abscissa followed by a strong flow deceleration towards the trailing edge [34]. This blade section has a stronger adverse pressure gradient than current designed LPT and has been widely studied through both experimental and numerical investigations. This LPT blade was experimentally investigated in the framework of the European project TATMo. The characteristics of the T106C cascade are summarized in Table 1. During the experimental investigations, different freestream turbulence intensities have been considered. The natural inlet turbulence intensity of the facility is $0.9 \%$. A passive turbulence grid was employed upstream of the cascade in order to generate distinct levels of free-stream turbulence intensity, from 1.8 to $3.2 \%$. In the present study, the natural turbulence intensity is only considered. The effect of freestream turbulence intensity was assessed by Benyahia et al. [15]. The Reynolds number $\operatorname{Re}_{2 \text { is }}$ based on the isentropic exit Mach number $\mathrm{M}_{2 \text { is }}$ and the blade chord $c$ ranges from 80000 to 250000 .

Rms quantities are a necessary condition to validate the LES results. Thus the choice of freestream turbulence intensity results also from the available experimental data. The averaged and rms values of the wall shear stress have only been measured at turbulence intensities of 0.9 and $1.8 \%$ [35]. Unfortunately this last level is still too low to be representative of real engine and there is no open bubble. It reinforces the choice of the natural turbulence intensity for which the bubble is open at Reynolds number $\operatorname{Re}_{2 \text { is }}$ equal to 80 000. It should be noticed that the wall shear layer is measured by surface-mounted hot-films which are insensitive to reversed flow.

Table $1:$ T106C main characteristics [35]

\begin{tabular}{|l|c|}
\hline Chord $c[\mathrm{~mm}]$ & 93.01 \\
\hline Pitch to chord ratio $g / c$ & 0.95 \\
\hline Aspect ratio $h / c$ & 2.40 \\
\hline Inlet flow angle $\beta_{1}\left[^{\circ}\right]$ & 32.7 \\
\hline Blade stagger $\gamma\left[^{\circ}\right]$ & 30.7 \\
\hline Isentropic exit Mach number $M_{2 i s}$ & 0.65 \\
\hline Diffusion factor & 0.42 \\
\hline
\end{tabular}

In experiments, the quasi wall shear stress is measured using hot-films sensors. The hot films sensors are insensitive to flow direction. Thus, the norm of quasi wall shear stress is only 
available. That's why only positive values are plotted. The hot-films sensors could not be calibrated and their output was processed in a semi quantitative manner [34][35]. Thus the charts show only the qualitative evolution of the quasi-wall shear stress. Therefore, the scale on each vertical axis should not be used to extrapolate quantitative values. Moreover the RMS value is relative to the voltage signal in experiments and to wall shear stress in CFD although the normalization is computed in a same manner i.e. using the time-averaged wall shear stress. It should be noticed that the numerical wall shear stress is computed using instantaneous velocity field allowing the computation of time-averaged and RMS values.

\section{Numerical methods}

The numerical simulations have been performed using the elsA software [36], developed at ONERA. This code is based on a cell centered finite volume technique and structured multiblock meshes. The viscous fluxes are computed with a second-order centered scheme. For efficiency, implicit time integration is employed to deal with the very small grid size encountered near the wall.

For LES computations, the convective fluxes are discretized with the third-order accurate AUSM+(P) scheme developed by Mary and Sagaut [37]. The numerical dissipation is proportional to the local fluid velocity. The time integration is performed with the second order backward scheme of Gear. The chosen time step is set equal to $10^{-7} \mathrm{~s}$. Therefore the CFL number is lower than 1 in the whole domain except in the boundary layer where its maximum value is 12. At each time step, an approximate Newton method is used to solve the non-linear problem using LU factorization method. Six Newton sub-iterations are used. The use of 6 sub-iterations per time-step is required to reach a decay superior to one and a half order of magnitude for the residuals. This criterion is a compromise between accuracy and cost of the computation and takes into account the small time step involved [38]. Moreover, Daude et al. [39] use a ratio of CFL/N equal to 2 where $\mathrm{N}$ is the sub-iteration number, to perform LES in order to reduce CPU cost in comparison to an explicit time integration scheme while the result quality is identical. In the present case, the maximum CFL is equal to 12. Thus 6 sub-iterations are used.

For the steady and unsteady RANS computations, the convective fluxes are computed with the second order upwind scheme of Roe [40] or the third-order AUSM+(P) scheme. The backward Euler time integration scheme has been employed. Local time step with a scalar LU-SSOR implicit method has been also used. As for LES, the time integration method of URANS simulations is the second-order scheme of Gear with a time step equal to $10^{-7} \mathrm{~s}$ and six sub-iterations. It is worth noting that the sensitivity of RANS results to numerical parameters is investigated in section 5.1.

In order to limit the size of the numerical mesh, the inlet plane of the computational domain is placed at half an chord upstream the leading edge, whereas, the outlet plane is at one and a half chord downstream the trailing edge to resolve the wake behind the blade. It is worth noting that the outlet plane is not used for the performance computations. The performance computations are based on data at upstream and downstream planes. The experimental data are specified at the inlet plane used as upstream plane, especially the total pressure used in loss computation, and the downstream plane is not the outlet plane of the computational domain but the outlet experimental plane which is located $0.55 \mathrm{C}_{\mathrm{ax}}$ downstream of the trailing edge. The numerical data are directly extracted in this plane and used for the performance computations. The computational domain consists of only one blade passage. The boundaries in pitchwise direction are assumed periodic. Because of the high aspect ratio $h / c$, the flow at midspan can be considered two dimensional, thereby allowing three-dimensional simulations to be performed under the assumption of a homogeneous flow in the spanwise direction. Thus the simulations are quasi-three dimensional with RANS modeling i.e. the meshes are 
composed of few points spanwise and a periodic condition is applied at the spanwise boundaries. For LES, the computational domain extends over $15 \%$ of chord spanwise which is a classical value [25][26][41] and the boundaries in spanwise and pitchwise directions are considered periodic. The LES computational domain is composed of 161 points in spanwise direction and is computed using a finite volume method, not a spectral one. For all computations, total enthalpy and pressure are specified at the inlet plane and a static pressure at the outlet plane while a no-slip adiabatic wall condition is applied at the blade surface.

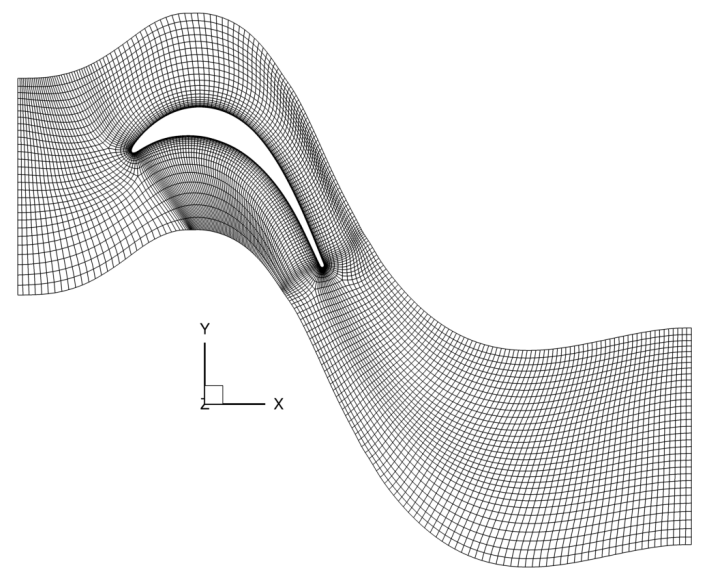

(a)

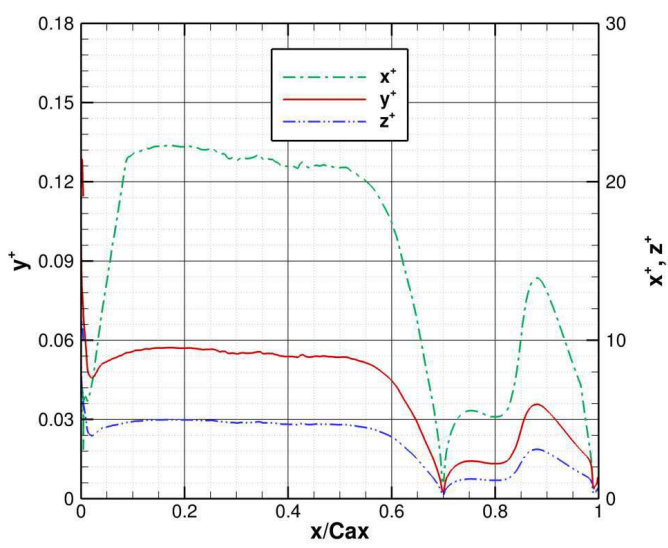

(b)

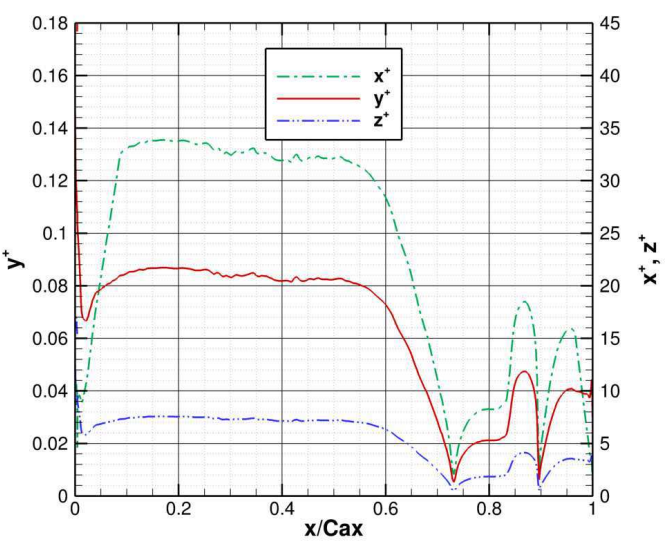

(c)

Figure 1: LES mesh with one out of 4 points in both streamwise and pitchwise directions (a) and $\mathrm{x}^{+}, \mathrm{y}^{+}$and $\mathrm{z}^{+}$distributions on suction side for $\mathrm{Re}_{2 \text { is }}=80000$ (b) and $\operatorname{Re}_{2 \text { is }}=140000$ (c)

The LES mesh is composed of 27 million points. This mesh appears to be refined enough to accurately describe the boundary layers developing on the blade. Figure 1a shows the computational domain at a given span (one out of four points in both streamwise and pitchwise directions) and the normalized dimensions of the cells on the wall are depicted in Figure $1 \mathrm{~b}\left(\operatorname{Re}_{2 \mathrm{is}}=80000\right)$ and Figure 1c $\left(\operatorname{Re}_{2 \mathrm{is}}=140000\right)$. The values relative to $\operatorname{Re}_{2 \mathrm{is}}=80$ 000 are lower than the required ones [42]: $50\left(\mathrm{x}^{+}\right), 1\left(\mathrm{y}^{+}\right)$and $15\left(\mathrm{z}^{+}\right)$. For the high computed Reynolds number $\left(\operatorname{Re}_{2 \text { is }}=140000\right)$, the normalized dimensions of the cells on the wall are higher than the required ones only at the leading edge. Three meshes have been used for RANS computations. The coarse, medium and fine meshes are respectively composed of 257 thousand, 0.9 million and 1.66 million points. The fine mesh is relative to the LES mesh with only eleven points spanwise. 


\section{Turbulence and transition modeling}

\subsection{RANS simulations}

The RANS computations are performed with the k- $\omega$ SST model of Menter [43]. Due to Boussinesq hypothesis, the Reynolds stress tensor immediately scales with the mean rate of strain tensor. It leads to very high turbulent production close to stagnation point and extra turbulence is convected downstream, deeply affecting the prediction. In the present case, for RANS simulations, the turbulent kinetic energy production term $\mathrm{P}_{\mathrm{k}}$ is limited using the turbulent dissipation such as $P_{k}=\min \left(2 v_{t} S_{i j} S_{i j} ; 10 \beta^{*} \omega\right)$.

As this turbulence model is unable to capture laminar-turbulent transition, this phenomenon is predicted with the transport equation model of Menter et al. [13][14]. This model has been implemented in elsA solver by Content and Houdeville [44] and Benyahia et al. [15]. All Reynolds numbers of the considered range are simulated with RANS model without and with transition model. For RANS computations, the turbulent Reynolds number $\mu \mathrm{t} / \mu$ is equal to 3 in the inlet plane. The turbulent Reynolds number value is chosen in order to have a good agreement of the turbulence decay from the inlet plane towards the airfoil with the experimental data. It is reminded that the freestream turbulence intensity at the leading edge is equal to $0.9 \%$. A preliminary study has been performed to determine these values at the current inlet plane. Figure 2 shows the experimental and CFD turbulence decay upstream of the leading edge. The experimental decay is extracted from [50]. The asymptotic turbulence decay obtained with CFD allows reaching the freestream of $0.9 \%$ at the leading edge. Using this law and the homogeneous isotropic turbulence decay, the turbulent dissipation $\varepsilon$ and then the turbulent Reynolds number can be estimated at the current inlet plane. The current $T u$ decay obtained by RANS simulations with transition model on the fine mesh fits the preliminary CFD result from the inlet plane to the leading edge.

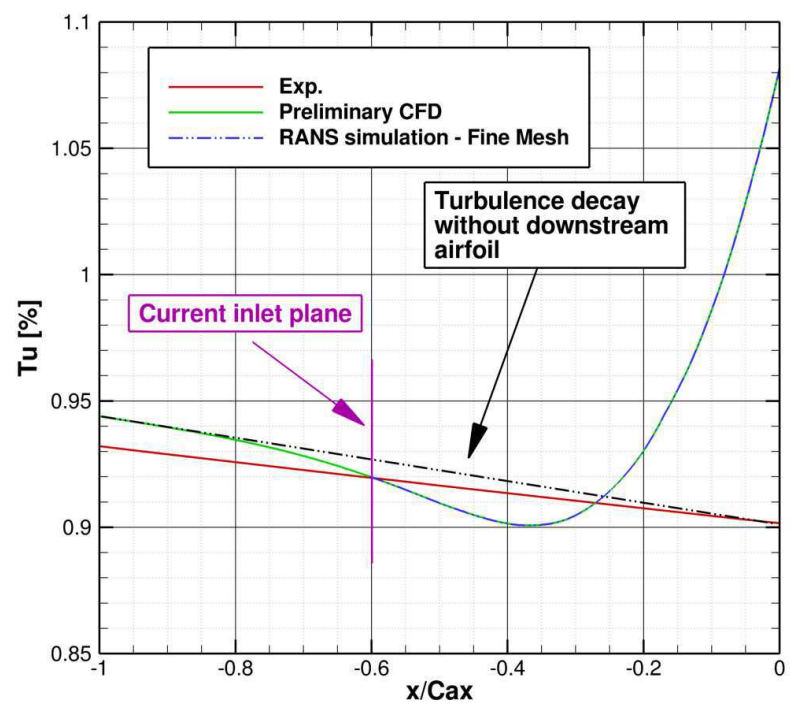

Figure 2: Experimental and CFD turbulence decay

The inlet values of turbulent kinetic energy and specific dissipation rate are computed from the freestream turbulence intensity and the inlet turbulent Reynolds number. Concerning the transition variables, the intermittency is set to 1.0 at the inlet of the computational domain and the momentum Reynolds number is set according to Langtry and Menter's correlation with $\lambda_{\theta}$ $=0.0[45]$. 


\subsection{Subgrid scale model}

The subgrid scale model (SGS) used in large-eddy simulation and chosen for the current investigation is the WALE model (Wall Adapting Local Eddy viscosity) proposed by Ducros et al. [46]. Nicoud and Ducros [47] show that a LES with the WALE SGS model does not produce any eddy viscosity in the case of wall-bounded laminar flow such as the Poiseuille flow. The amount of turbulent diffusion would be negligible and the development of linearly unstable waves would be possible. As the Smagorinsky invariant is large in pure shear layer, the Smagorinsky SGS model is unable to capture the laminar-turbulent transition. In the present study, the zero production of eddy viscosity was checked. Figure 3 depicts the turbulent (subgrid) over molecular viscosity ratio. Lines represent isovalue of normalized spanwise vorticity. In the laminar boundary layer, the subgrid viscosity is six times smaller than the molecular viscosity. So the turbulent viscosity is very close to zero. The turbulent viscosity production begins sufficiently far downstream of the separation point, near the transition point.

Calling the cell volume $\Delta$, the subgrid viscosity $\mu_{\mathrm{sg}}$ is defined as

$$
\mu_{s g}=(C \Delta)^{2} \frac{\left(S_{i j}^{d} S_{i j}^{d}\right)^{3 / 2}}{\left(\overline{S_{i j} S_{i j}}\right)^{5 / 2}+\left(S_{i j}^{d} S_{i j}^{d}\right)^{5 / 4}}
$$

With

$$
S_{i j}^{d}=\frac{1}{2}\left({\overline{g_{i j}}}^{2}+{\overline{g_{j i}}}^{2}\right)-\frac{1}{3}{\overline{g_{k k}}}^{2} \delta_{i j} ; \overline{g_{i j}}=\frac{\partial \overline{u_{i}}}{\partial x_{j}} \text { and } C=0.5
$$

The constant $C$ of this model is equal to 0.5. LES computations concern only two Reynolds numbers: $\operatorname{Re}_{2 \text { is }}=80000$ and $\operatorname{Re}_{2 \text { is }}=140$ 000. For $\operatorname{Re}_{2 \text { is }}=80000$, two simulations are performed, without and with freestream turbulence. The generation of random turbulent flow at the inlet boundary is detailed in the next section.

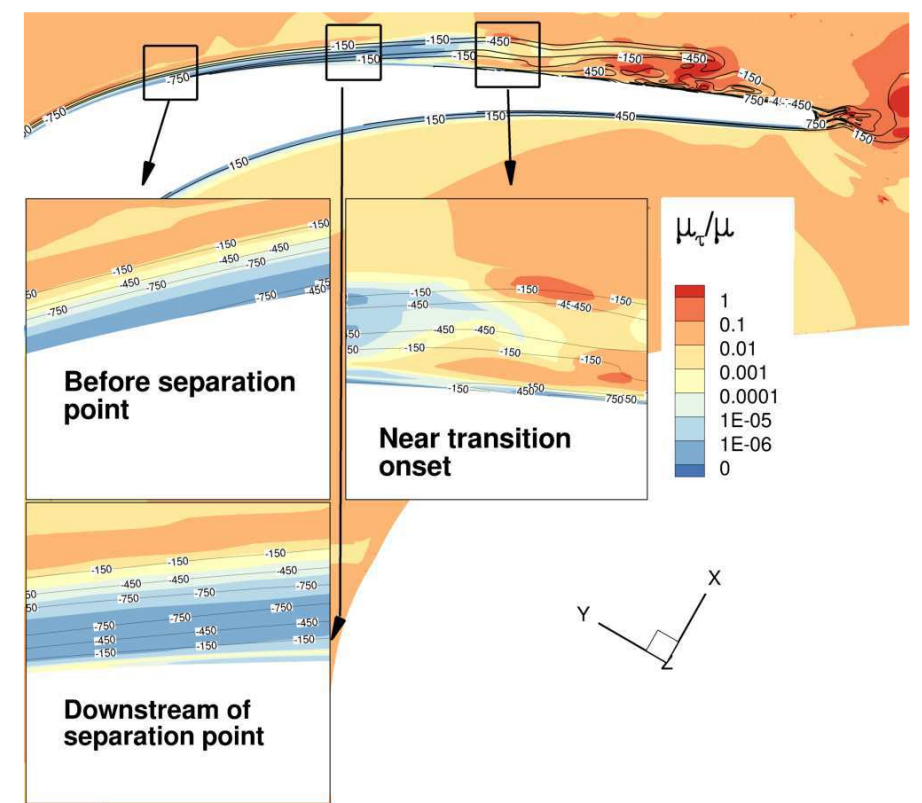

Figure 3: Turbulent over molecular viscosity ratio at an arbitrary time. 


\subsection{Turbulent random flow generation technique}

The generation of random turbulent flow at the inlet boundary is performed with the technique developed by Smirnov et al. [1]. Recent studies show the benefits of this method for LES and zonal hybrid RANS/LES computations [18][48]. Brunet [48] has integrated the random flow generation technique developed by Smirnov et al. [1] (RFG) into the elsA solver of ONERA [36]. The aim of the method is to determine the perturbation velocity relative to the targeted averaged turbulence. The first step consists in imposing the Reynolds stress tensor corresponding to this averaged turbulence.

$$
\tau_{i j}^{R}=\overline{u_{i}^{\prime} u_{j}^{\prime}}
$$

This problem with six unknowns is simplified to a three-unknown system $c_{n}$ using the orthogonal transformation tensor $a_{i j}$ that would diagonalize $\tau_{i j}{ }^{R}$ :

$$
a_{m i} a_{n j} \tau_{i j}^{R}=\delta_{m n}\left(c_{n}\right)^{2}
$$

The coefficients $c_{n}$ play the role of turbulent fluctuating velocities $\left(u^{\prime}, v^{\prime}\right.$ or $\left.w^{\prime}\right)$ in the new coordinate system produced by the transformation tensor $a_{i j}$. As this approach is an evolution of the Kraichnan's technique [49], it is based on a superposition of harmonic functions to generate a perturbation velocity vector:

$$
v_{i}\left(x_{i}, t\right)=\sqrt{\frac{2}{N}} \sum_{n=1}^{N}\left[p_{i}^{n} \cos \left(\tilde{k}_{i}^{n} \tilde{x}_{i}+\omega_{n} \tilde{t}\right)+q_{i}^{n} \sin \left(\tilde{k}_{i}^{n} \tilde{x}_{i}+\omega_{n} \tilde{t}\right)\right]
$$

With

$$
\begin{gathered}
\tilde{k}_{i}^{n}=k_{i}^{n} \frac{c}{c_{i}} ; \tilde{x}_{i}=\frac{x_{i}^{n}}{l} ; \tilde{t}=\frac{t}{\tau} ; c=\frac{l}{\tau} \\
p_{i}^{n}=\varepsilon_{i j m} \varsigma_{j}^{n} k_{m}^{n} ; q_{i}^{n}=\varepsilon_{i j m} \xi_{j}^{n} k_{m}^{n} ; \zeta_{j}^{n}, \xi_{j}^{n}, \omega_{n} \in N(0,1) ; k_{i}^{n} \in N\left(0, \frac{1}{2}\right)
\end{gathered}
$$

Where $l, \tau$ are the length- and the time-scales of turbulence, $\varepsilon_{\mathrm{ijk}}$ is the permutation tensor used in vector product operation and $N(M, \sigma)$ is a normal distribution of $N$ samples with mean $M$ and standard deviation $\sigma$. These random perturbation variables are generated only once for an entire simulation, which ensures a spatial and time coherency for the different blocks of the simulation. It could be noticed that the numbers $\mathrm{k}_{\mathrm{j}}{ }^{\mathrm{n}}$ and $\omega_{\mathrm{n}}$ represent a sample of $n$ wavenumber vectors and frequencies of the modeled turbulence spectrum:

$$
E(k)=16\left(\frac{2}{\pi}\right)^{\frac{1}{2}} k^{4} \exp \left(-2 k^{2}\right)
$$

Finally, the perturbation vector is given by:

$$
w_{i}\left(x_{i}, t\right)=c_{i} v_{i}\left(x_{i}, t\right) \text { and } u_{i}^{\prime}\left(x_{i}, t\right)=a_{i k} w_{k}\left(x_{i}, t\right)
$$

The considered boundary conditions chosen to apply this perturbation method impose to fix the total pressure, the total enthalpy, the normal unity velocity vector. It respects the 
characteristic theory for subsonic boundary conditions. To maintain this, the turbulent perturbation imposed thanks to the RFG technique is introduced through local modifications of the three previous imposed physical parameters of the boundary condition. This technique requires the Reynolds Stress tensor, characteristic turbulence length and time scales and the number of random samples. It allows generating non-homogeneous anisotropic flow field. Smirnov et al. [1] validated the turbulent random flow generation technique with isotropic homogeneous turbulence, anisotropic homogeneous turbulence and anisotropic nonhomogeneous turbulence. All these characteristics are well captured by this method. They also generate the turbulent field behind a flat plate. The technique is able to reproduce the turbulent characteristics of the flat plate wake as the inertial range. Although the turbulence decay is well captured, they consider that better results are expected using a finer grid. Brunet [48] studied the influence of the turbulent length scale on the generated turbulence and its advection. After a rapid decrease in the first cells downstream of the inlet boundary condition due to the damping of non-physical fluctuations, the turbulence level is quite stable even if the turbulent diffusion increases for smaller length scale. Nevertheless, if the boundary conditions are generated using realistic turbulence quantities and spectra, the transition region is small [1].

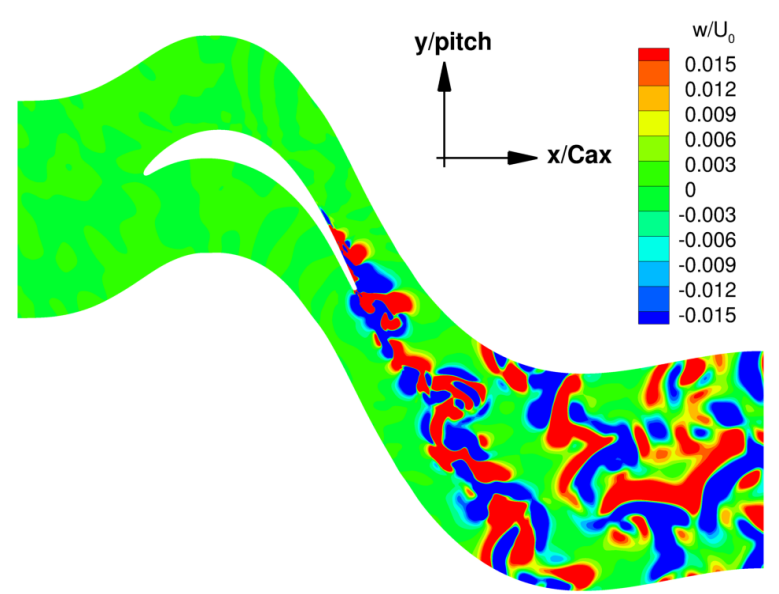

(a) Without freestream turbulence

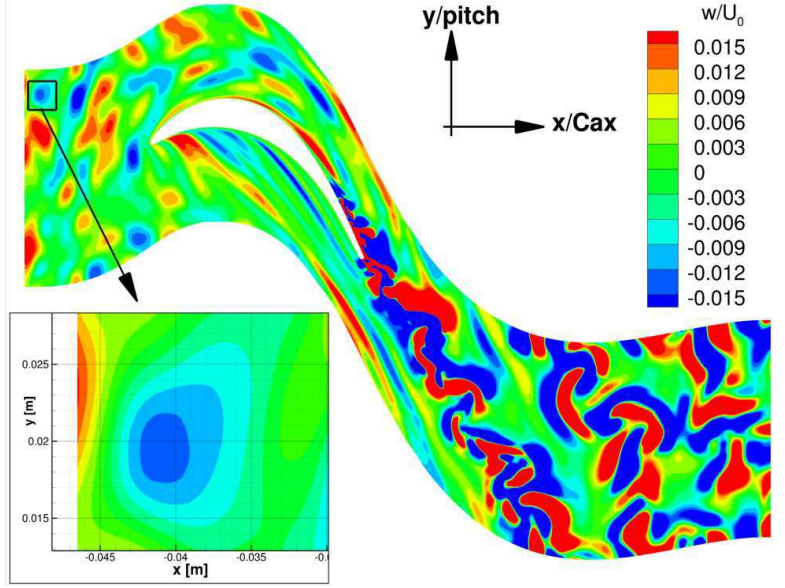

(b) With freestream turbulence Smirnov's method

Figure 4: Instantaneous spanwise velocity distribution at midspan - Without (a) and with (b) freestream turbulence $-\mathrm{Re}_{2 \mathrm{is}}=80000$

In practice, it requires the turbulent rate $T u$ and the turbulent length scale $l$. In the present case, $T u$ is equal to $0.9 \%$ and the length scale is equal to the experimental integral scale [35]. Its value is $4 \mathrm{~mm}$ and is equal to $29 \%$ of the spanwise extension of the computational domain and $4.5 \%$ of the pitchwise one. Figure 4 depicts the instantaneous spanwise velocity at midspan for $\mathrm{Re}_{2 \text { is }}=80$ 000. The comparison of the distributions shows the injection of coherent structures relative to the freestream turbulence. As the average spanwise velocity is equal to zero upstream of the blade, the fluctuating part is directly the instantaneous value. The levels of fluctuating spanwise velocity observed in Figure $4 \mathrm{~b}$ are coherent with the turbulent velocity scale $v=\frac{l}{\tau}$ normalized by freestream velocity $\mathrm{v} / \mathrm{U}_{0}=7 \mathrm{e}-3$.

As shown by equations relative to the Smirnov method, the injected freestream turbulence is a noise distributed along with a normalized length which is equal to 1 . The target length scale is then used to have a distribution around it. So, contrary to Synthetic Eddy Method, all eddies do not have the same characteristic length. However, this length comes from the previous distribution. It is expected to have eddy length slightly smaller or higher than the targeted length $(4 \mathrm{~mm}$ in the present case). Figure $4 \mathrm{~b}$ shows that the size of a freestream eddy 
described by a blue patch is close to $4 \mathrm{~mm}$, especially before the eddy stretching due to the velocity field in the vicinity of the airfoil and its leading edge.

The freestream turbulence intensity is computed for URANS and LES simulations. From the inlet plane, as the distance to the leading edge decreases, the velocity decreases and the turbulent kinetic energy decreases due to the turbulence decay. Nevertheless, close to the leading edge, the turbulent kinetic energy increased. It results in a decrease followed by an increase of the turbulence intensity. Very close to the leading edge, the turbulence intensity is close to $1 \%$ for both URANS and LES.

\section{RANS and time-averaged LES results}

\subsection{Performances}

Figure 5 shows the midspan isentropic Mach number distributions along the blade for three Reynolds numbers $\operatorname{Re}_{2 \text { is }}$. For all considered Reynolds numbers boundary layer separation occurs on the suction side in the adverse pressure gradient region. Separation is well visible on the isentropic Mach number plots: a small to long plateau appears in the decelerating region. The experimental data and the CFD results are in very good agreement. The isentropic Mach number peak is well captured as well as the separation point except for the lowest Reynolds number $\operatorname{Re}_{2 \text { is }}=80000$ (figures Figure $5 \mathrm{a}$ and Figure $5 \mathrm{~b}$ ) for which the separation point is predicted downstream of the experimental separation point (cf. section 5.2). For the case $\mathrm{Re}_{2 \text { is }}=140000$, the reattachment point is located upstream and downstream of the experimental point respectively for RANS and LES results. As shown in the next section, the discrepancy is close to $6 \%$ of axial chord $\mathrm{C}_{\mathrm{ax}}$. For all Reynolds number, the fully turbulent RANS simulations do not predict any separation or bubble. The mesh density effect is clearly visible between the coarse and the medium mesh, especially for $\operatorname{Re}_{2 \text { is }}=80000$. As the number of points is increased, the separation point moves downstream. As shown in section 5.3, the simulation based on the coarse mesh predicts an open bubble while a long bubble is captured by the medium and fine meshes. The discrepancy between the medium and the fine mesh is sufficiently small to assume that the mesh convergence is almost reached. Babajee and Arts [50] show a similar effect of mesh on isentropic Mach number distribution of the T108 blade: the prediction is improved if the wake is well taken into account in the mesh generation process, resulting in a reattachment point located upstream of the point predicted on a coarse mesh. This result is consistent with the present study. The numerical scheme has also an effect on isentropic Mach number distribution with the fine mesh but it is very small on the separation and pressure recovery points. For all Reynolds numbers, steady and timeaveraged unsteady RANS results are very similar. It points out that the unsteadiness captured in unsteady RANS simulation has small amplitude. This assumption will be investigated later. 


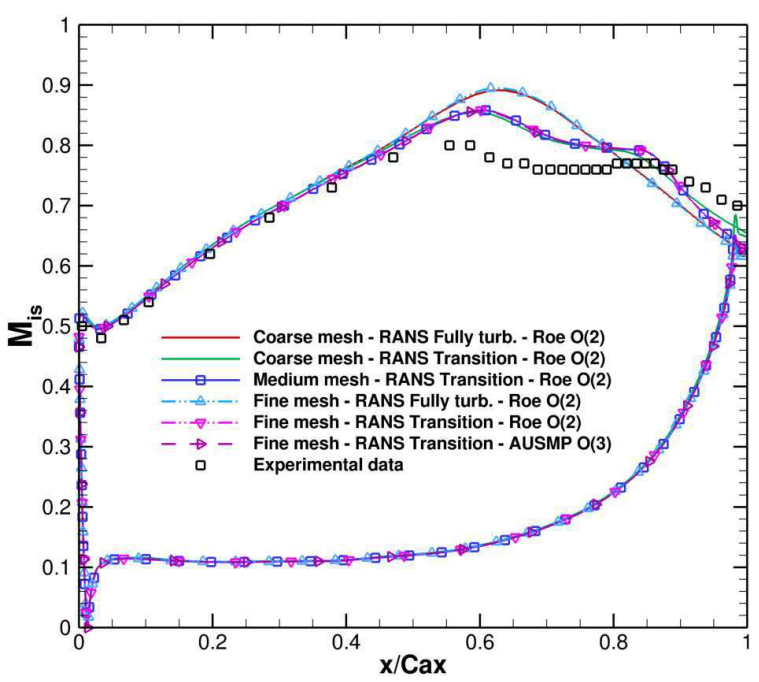

(a) $\mathrm{Re}_{2 \mathrm{is}}=80000$ - Steady RANS results

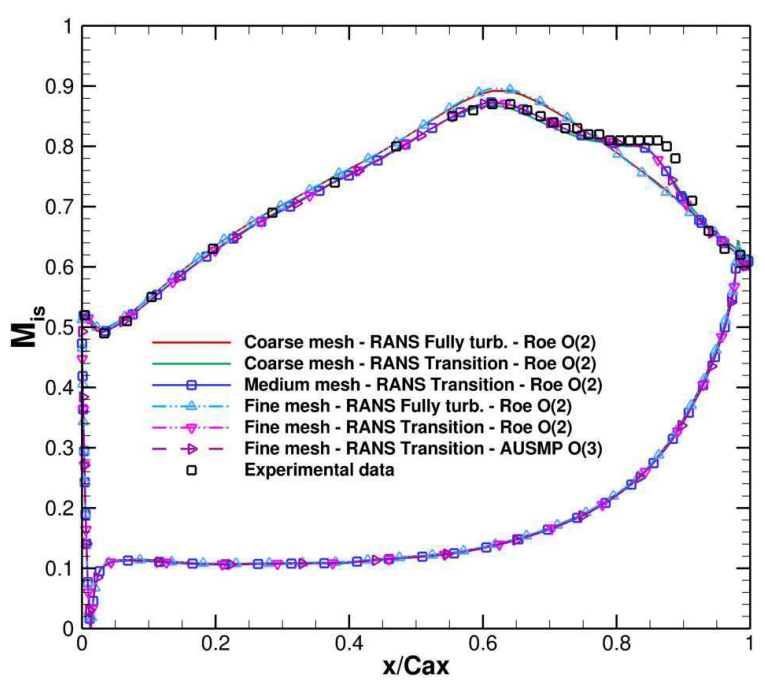

(c) $\mathrm{Re}_{2 \mathrm{is}}=140000$ - Steady RANS results

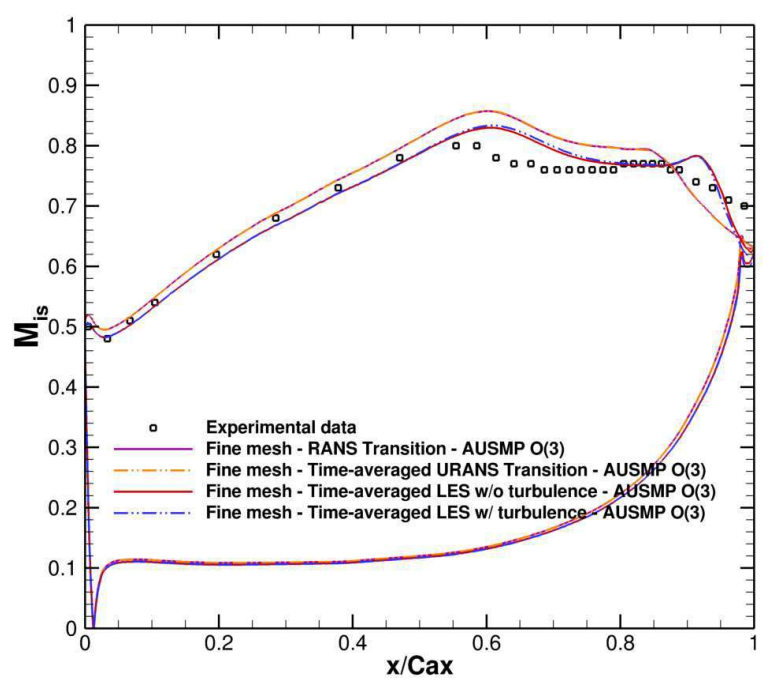

(b) $\mathrm{Re}_{2 \mathrm{is}}=80000$ - RANS, URANS and LES results

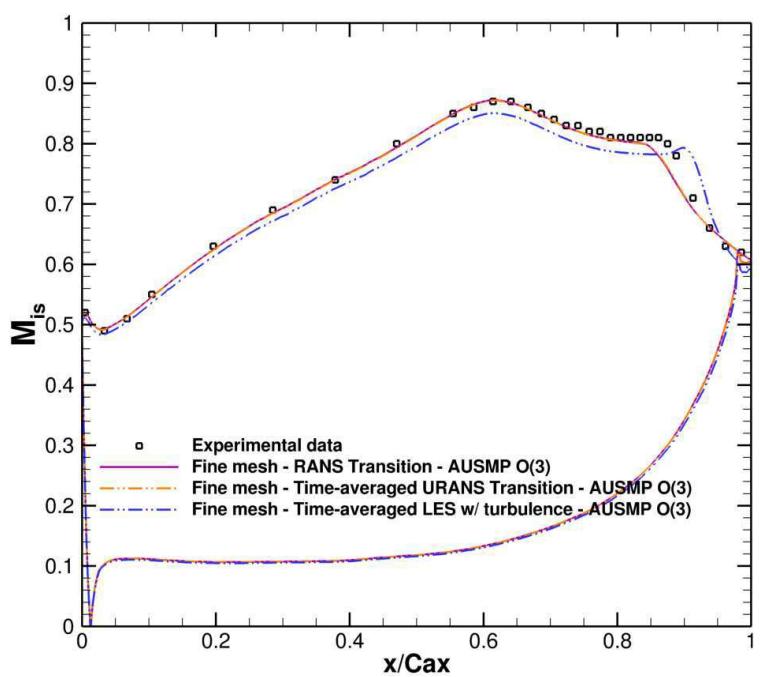

(d) $\mathrm{Re}_{2 \mathrm{is}}=140000-$ RANS, URANS and LES results

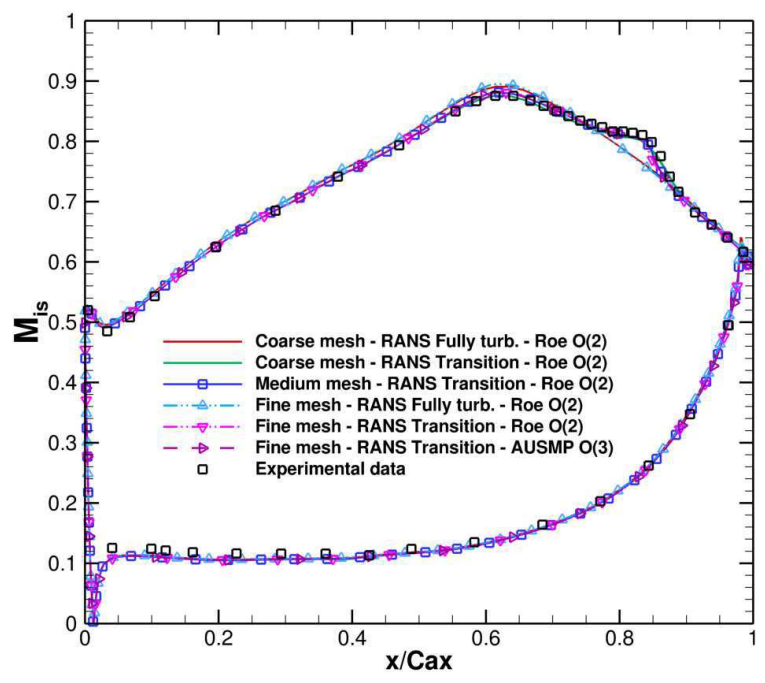

(e) $\operatorname{Re}_{2 \text { is }}=250000$ - Steady RANS results

Figure 5: Isentropic Mach distribution on T106C blade at midspan for three different isentropic exit Reynolds number 
The main difference with experimental data concerns the case $\operatorname{Re}_{2 \text { is }}=80$ 000: the predicted bubble is not open which explains the discrepancy near the trailing edge. In the separated flow region, the isentropic Mach number distributions obtained numerically are close to the ones obtained experimentally, at least for the level because the main discrepancy comes from the separation point location. A decrease of the Reynolds number leads to an increase of the pressure plateau size which corresponds to a growth of the laminar separation bubble. For all Reynolds number, the isentropic Mach number of LES is computed from the time-averaged flow. The LES improves the isentropic Mach number distribution although the separation point is predicted downstream of the experimental one. The isentropic Mach number level is closer to the experimental data, especially in the vicinity of the plateau. The comparison of isentropic Mach number predicted by LES without and with freestream turbulence shows that this incoming turbulence has a small effect on results. The separation point is slightly moved downstream with freestream turbulence: the normalized curvilinear abscissa which is equal to zero at the leading edge and one at the trailing edge traveling the suction side is increased by 0.005 (cf. section 5.2). This is due to the generation of small fluctuations in the laminar boundary layer which allows a higher ability to overcome the adverse pressure gradient. As the freestream turbulence intensity is small, the effect of turbulence is limited.

For the case $\mathrm{Re}_{2 \mathrm{is}}=140$ 000, the RANS simulations with transition modeling predict an isentropic Mach number close to the experimental data, except for the reattachment point. This is due to the prediction of a small laminar separation bubble for this Reynolds number and the prediction of the bursting for a lower Reynolds number. Thus the effect of the bubble on the pressure distribution is smaller for RANS simulations and hence the plateau length is smaller for RANS simulations with respect to experimental data. The isentropic Mach number distribution obtained with time-averaged LES with freestream turbulence is close to the experimental data although the level is smaller than the experimental one. Contrary to the RANS simulations, the LES simulation predicts a reattachment point downstream of the experimental one. As shown in section 5.3, the LES simulation captures a long bubble which explains that the laminar separation bubble length is slightly higher for the LES simulation than the experimental one. For RANS simulations with transition modeling and LES simulation, the pressure recovery is well predicted.

For the highest computed Reynolds number $\operatorname{Re}_{2 \mathrm{is}}=250000$, the steady and time-averaged unsteady RANS predictions are close to the experimental data. The short bubble is wellcaptured by CFD. The mesh density has a small effect between the coarse and the medium mesh. The difference due to the increase of mesh density from the medium to the fine mesh and to the numerical schemes is almost negligible.

Similar RANS results are found in open literature. The isentropic Mach number distribution obtained with TRACE solver of DLR [51] is close to the present predictions, even if there is no low Reynolds study as the lowest computed Reynolds number is 120 000. Corral and Gisbert [52] show a better agreement with experimental data in comparison to the present predictions, especially at the lowest Reynolds number. The investigations of Babajee and Arts [50] and Pacciani et al. [53] show similar distributions, especially at the lower Reynolds number $\left(\operatorname{Re}_{2 \mathrm{is}}=80\right.$ 000). As shown by Babajee and Arts (Figure 9 in [50]), the injected turbulence parameters have a strong influence on laminar separation bubble prediction. At the lowest Reynolds number, using the wind tunnel decay law, the bubble is not correctly predicted while with a smaller turbulent Reynolds number, the bubble is longer and loss are closer to experimental data. However, at a higher Reynolds number, only the wind tunnel parameters lead to a correct prediction of loss. Thus the influence of the turbulent Reynolds number specified at the boundary condition has a great influence on results, probably much more than the calibrated functions. It should be noticed that current RANS isentropic Mach number distributions are close to their results (Figure 12 in [50]). In [53], the isentropic Mach number distributions at Re2is $=80000$ obtained with Langtry-Menter and Content- 
Houdeville functions are closed to those obtained in the present investigation. Pacciani et al. obtained results in a better agreement with experimental data with the LKE model. However, the authors use the turbulent length scale over axial chord ratio equal to $2.5 \mathrm{e}-3$. With an axial chord close to $0.08 \mathrm{~m}$, this turbulent length scale is equal to $0.2 \mathrm{~mm}$ instead of $4 \mathrm{~mm}$ [35]. Thus the turbulent Reynolds number which is proportional to $l \sqrt{k}$, is 20 times smaller than the experimental one. The work of Babajee and Arts shows that a small value of turbulent Reynolds number leads to a slight better agreement in isentropic Mach number distribution. From these works, the discrepancy with the experimental data can be explained by:

- The correlation functions in transition model of Menter et al. [13][14]. In these publications, two functions were not published: $F_{\text {length }}$ and $R_{\theta C}$. Different authors have proposed correlations [45][44][52]. Pacciani et al. [53] show that significant different predictions are obtained using various correlation functions.

- The diffusion constant of the transition model. Two values are generally used: 2 [45] and 10 [15]. Babajee and Arts [50] shows that this parameter has a strong effect on isentropic Mach number distribution and subsequently on losses.

- The applied boundary condition for the turbulent quantities. The freestream turbulence level and turbulent Reynolds number $R e_{t}$ have to be carefully specified. In the present study, these parameters are equal to $0.9 \%$ and 3 (cf. section 3). Babajee and Arts [50] exhibit an important influence of the turbulent Reynolds number $R e_{t}$ on the isentropic Mach number distribution and losses, especially at the lowest Reynolds number $\operatorname{Re}_{2 \mathrm{is}}$ $=80000$ where an open bubble can be predicted if the turbulent Reynolds number $R e_{t}$ is small i.e. close to 0.01. In the present study, the turbulent Reynolds number value is chosen in order to have a good agreement of the turbulence decay from the inlet plane towards the airfoil with the experimental data.

In the present investigation, the transition point error is directly linked to the separation point error. The separation error is mainly driven by inflow and outflow boundary conditions which have been calibrated at the higher Reynolds number (250 000) with RANS simulations and by the numerical scheme which could be too dissipative despite the third order.

For the LES predictions, the discrepancies with the experimental data can also be explained by the sub-grid scale model (WALE in the present study), the filter induced by the mesh, the discretization of the shear layer region and the spanwise extension of the domain [21]. If the number of points is not sufficiently high in the shear layer, the instability cannot be wellpredicted and can be delayed. Moreover, the very small delay in transition can be due to the span length which influences the development of two- and then three-dimensional instability. Due to the computational cost of LES, no parametric investigation has been performed in order to assess the influence of these parameters on transition prediction. As most of LES of T106 blade concern either the T106A airfoil or the low-speed tests in open literature, it is difficult to compare quantitatively the current results to investigations of other authors. Nevertheless, a discrepancy on the time-average static pressure field on the suction side is observed in Raverdy et al. [20], Ooba et al. [54], Matsuura and Kato [22] and Funazaki et al. [23] by comparison to experimental data or DNS results. 

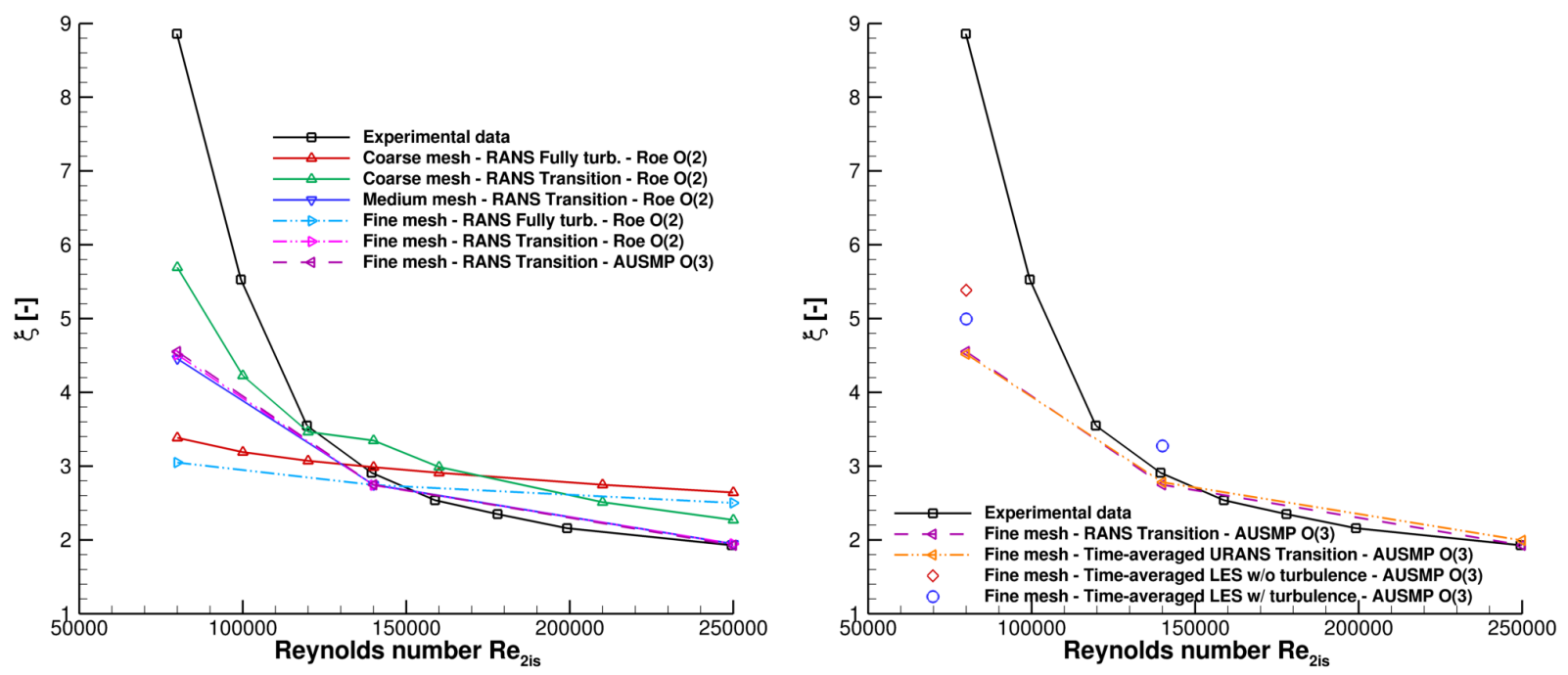

Mass-weighted kinetic energy losses - RANS simulations

Mass-weighted kinetic energy losses URANS and LES simulations

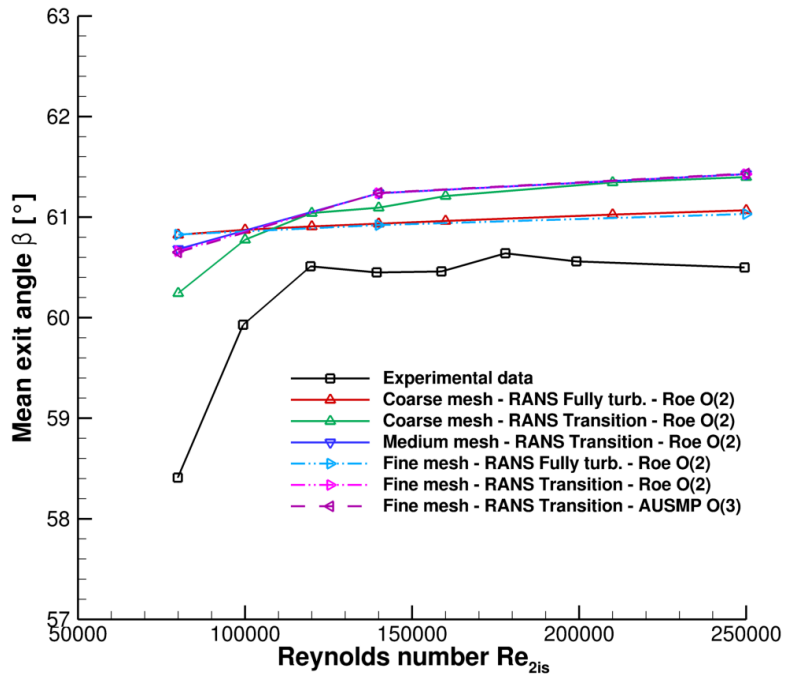

(a) Mass-weighted outlet flow angle RANS simulations

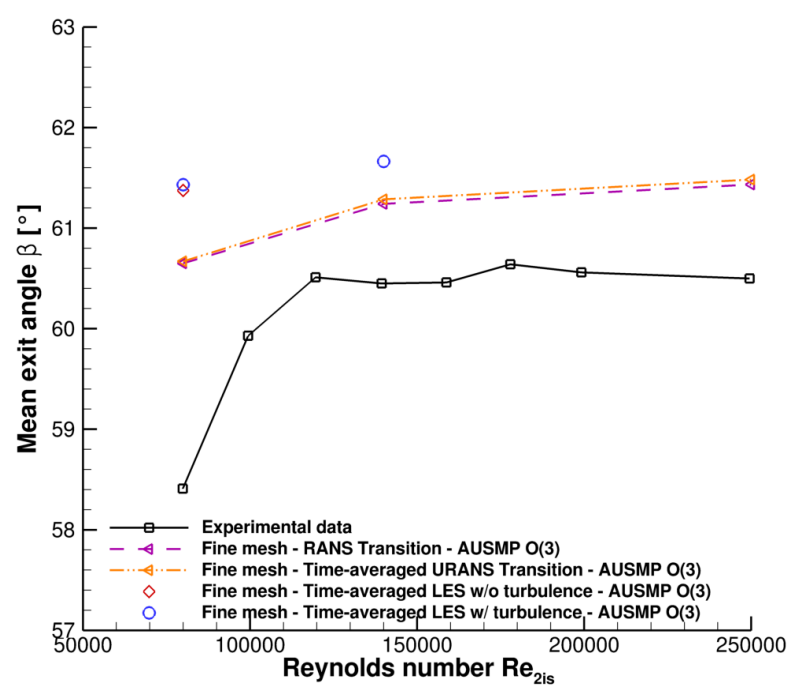

(d) Mass-weighted outlet flow angle URANS and LES simulations

Figure 6: Mass-weighted kinetic energy losses (a-b) and mass-weighted outlet flow angle (cd) as a function of $\operatorname{Re}_{2 \text { is }}$

The weak cost of RANS computations allows performing studies about the effect of Reynolds number and of mesh density. Figure $6 \mathrm{a}$ and Figure $6 \mathrm{~b}$ depict the mass-weighted kinetic energy loss coefficient which is defined as:

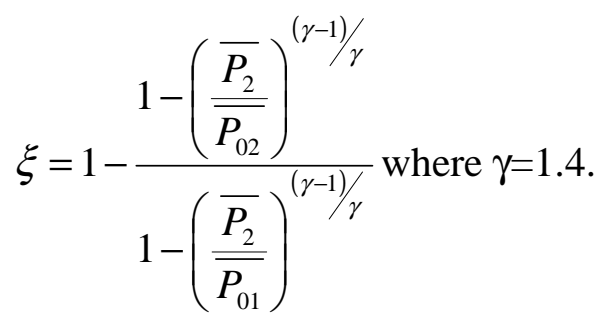

The fully turbulent simulations are unable to capture the evolution of the mass-weighted kinetic energy loss coefficient with respect to isentropic exit Reynolds number. At higher Reynolds number, the losses are higher than the experimental one due to the friction of the fully turbulent boundary layer while the RANS computation with transition model leads to a 
value closer to the experimental data because of the laminar part of the boundary layer and of the short laminar separation bubble. At lower Reynolds number, due to the fully turbulent boundary layer, there is no separation. So the losses are smaller than the experimental one. On the contrary, the RANS simulations with transition model are able to capture the variation of the laminar separation bubble with the Reynolds number. At low Reynolds, as the bubble is open or at least long, the losses are high and closer to experimental data. Moreover the influence of the mesh density is clearly important. The finer the mesh is, the lower is the numerical dissipation. Thus the mass weighted kinetic energy loss coefficient is lower for the fine mesh. The mesh effect is higher for the lowest Reynolds number because the flow is very sensitive to the numerical dissipation, especially the laminar separation bubble. The influence of the mesh density is amplified by the discrepancy in bubble type. The predicted bubble is open for the coarse mesh while it is long for the fine mesh. The small discrepancy between the predictions on medium and fine meshes reinforces the idea that the mesh convergence is almost reached for RANS simulations. The spatial discretization (second order scheme of Roe vs. third order scheme AUSM+(P)) and time integration (steady vs. unsteady) schemes have not significant impact on prediction on the fine mesh. In comparison to the RANS results obtained on the fine mesh, the LES simulation improves the prediction of losses for the lowest Reynolds number $\left(\operatorname{Re}_{2 \mathrm{is}}=80000\right)$ because the laminar separation bubble is longer and thicker for LES simulations. As the bubble is thinner with freestream turbulence, the losses are smaller for this case. For the case $\operatorname{Re}_{2 \mathrm{is}}=140$ 000, as the LES simulation over predicts the bursting Reynolds number, the losses are higher than the experimental ones. Despite the high discrepancy with experimental data at the lowest Reynolds number, LES results are closer to experimental data than RANS results even with transition modeling. However, the discrepancy is too high to have a predictive numerical tool.

Figure $6 \mathrm{c}$ and Figure $6 \mathrm{~d}$ show the evolution of the mass-weighted outlet flow angle with respect to isentropic exit Reynolds number. In the experiment, this angle is quite constant as long as the bubble is short or long. The angle drops when the bubble becomes open. This variation is only captured by the steady RANS simulation on the coarse mesh as the predicted bubble is open. Nevertheless, the angle is overestimated by $1.8^{\circ}$ at the lowest Reynolds number. As the bubble is long in the RANS predictions on the medium and fine meshes, the drop is not well-captured and the angle is even more overestimated $\left(2.3^{\circ}\right)$. The LES results are not in good agreement with the experimental data. It results from the inability to capture the open bubble and from the wake widening caused by vortex ejection during the transition process (cf. section 6.1). Thus the pitchwise and streamwise components of velocity are respectively higher and smaller than the RANS and probably even more than the experimental data. Moreover, three-dimensional effects could also explain the discrepancy between numerical results and experimental data.

As the mesh convergence is almost reached and the numerical parameters have a small effect, only the steady and unsteady RANS simulations based on the fine mesh and the third-order scheme AUSM+(P) are kept for the following sections.

\subsection{Flow topology parameters}

Figure $7 \mathrm{a}$ and Figure $7 \mathrm{c}$ depict the evolution of the time-averaged wall shear stress and its rms value along the blade suction side for $\operatorname{Re}_{2 \text { is }}=80000$. Downstream of the peak of velocity, due to the flow deceleration, the wall shear stress decreases rapidly and tends towards zero. The presence of separation bubble is indicated by the region where the time-averaged wall shear stress is close to zero. The separation point is observed near $\mathrm{x} / \mathrm{C}_{\mathrm{ax}}=0.64$ while this one is predicted more downstream by the simulations. The steady and time-averaged unsteady RANS simulations predict the separation around $\mathrm{x} / \mathrm{C}_{\mathrm{ax}}=0.7$ and the LES simulations around $\mathrm{x} / \mathrm{C}_{\mathrm{ax}}=0.71$. The freestream turbulence level has a small influence on the location of this point. Without any freestream turbulence, the separation is slightly upstream: $x / C_{a x}=0.71 \mathrm{vs}$. 
$\mathrm{x} / \mathrm{C}_{\mathrm{ax}}=0.715$. Whatever the data (experimental or numerical), the separation point is located where the descending slope of the velocity distribution, such as the isentropic Mach number distribution, starts to reduce its inclination (Figure 5a and Figure 5b).

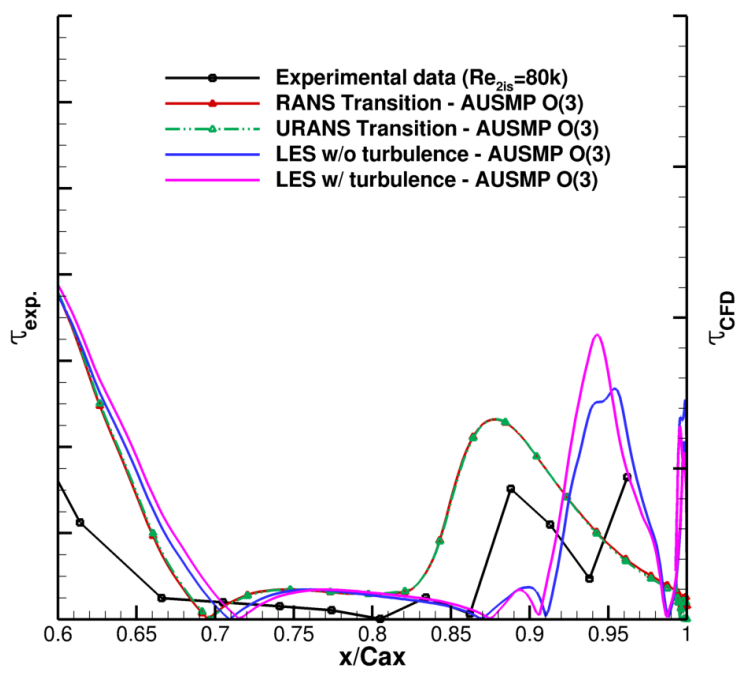

(a) $\mathrm{Re}_{2 \mathrm{is}}=80000$ - Steady or timeaveraged wall shear stress

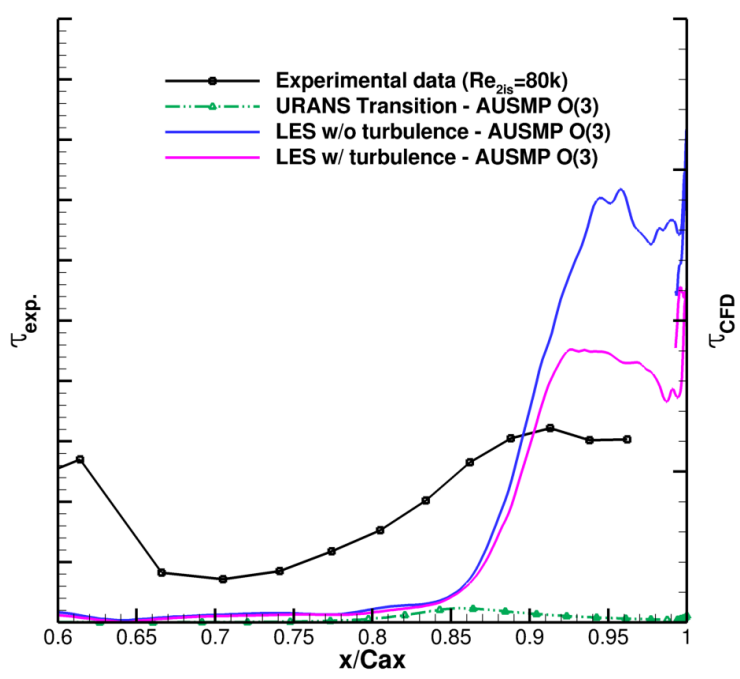

(c) $\operatorname{Re}_{2 \mathrm{is}}=80000-\mathrm{RMS}$ values of wall shear stress

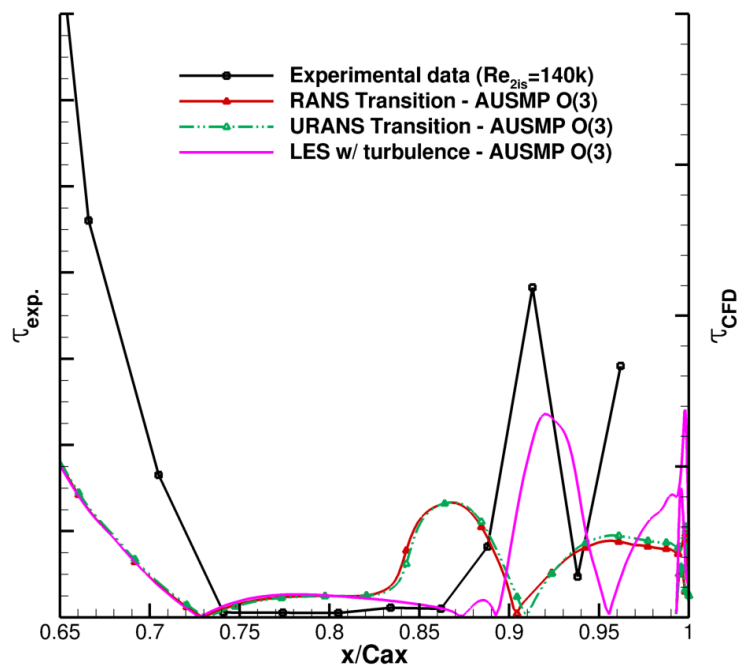

(b) $\mathrm{Re}_{2 \mathrm{is}}=140000$ - Steady or timeaveraged wall shear stress

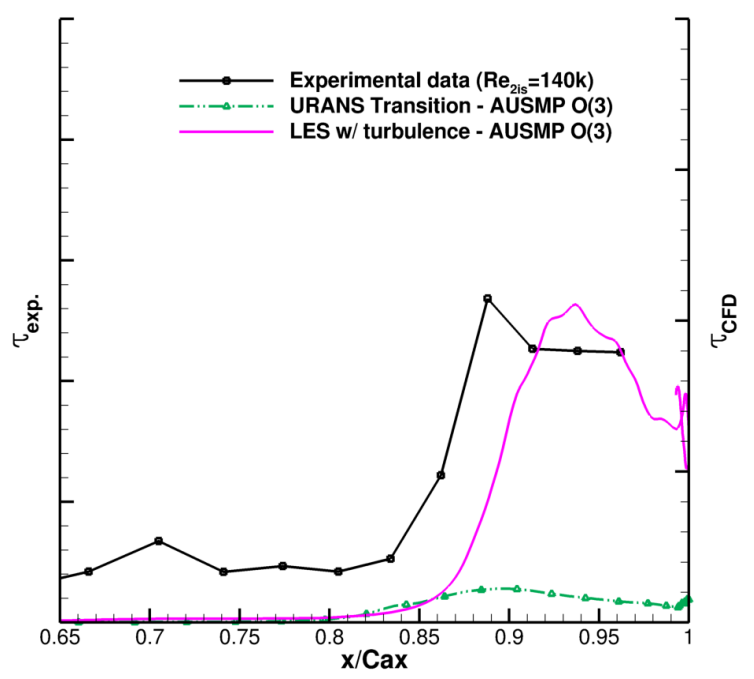

(d) $\mathrm{Re}_{2 \mathrm{is}}=140000$ - RMS values of wall shear stress

Figure 7: Wall shear stress distribution on T106C blade at midspan for two different isentropic exit Reynolds numbers

The transition point can be deduced from the rms value of wall shear stress: the onset is located at the rise beginning. Thus the experimental transition point is located at $\mathrm{x} / \mathrm{C}_{\mathrm{ax}}=0.74$ and once again the CFD points are downstream of the experimental one: $\mathrm{x} / \mathrm{C}_{\mathrm{ax}}=0.8$ for the unsteady RANS simulation and $\mathrm{x} / \mathrm{C}_{\mathrm{ax}}=0.85$ for both LES computations. This difference is similar to results of Michelassi et al. [25] where the transition point is delayed by $0.1 \mathrm{C}_{\mathrm{ax}}$ due to filtered frequencies. As shown previously, steady and time-averaged RANS predictions are very similar. One could assume that the transition onset predicted by steady RANS computation is close to the time-averaged unsteady RANS prediction i.e. $\mathrm{x} / \mathrm{C}_{\mathrm{ax}}=0.8$. The experimental transition point is located in the middle of the pressure plateau as for the LES (Figure 5b). Nevertheless, the RANS simulations predict a transition point at the plateau beginning (Figure 5a). 
After laminar-turbulent transition, one reaches the pressure recovery point following by the reattachment point. As the transition location differs from experimental and CFD results, a discrepancy is still observed for the pressure recovery point. It explains the shift on the wall shear stress evolution. As the wall shear stress is unable to distinguish the flow direction, the pressure recovery and reattachment points are difficult to locate using the criteria of wall shear stress increase. In the present study, for CFD results, the streamlines around the blade airfoil are used (Figure 9) for the last point. The reattachment point is located at $\mathrm{x} / \mathrm{C}_{\mathrm{ax}}=1.0$ for steady and time-averaged unsteady RANS computations and $\mathrm{x} / \mathrm{C}_{\mathrm{ax}}=0.99$ for LES results. As there is an open bubble in experiment, the pressure recovery and reattachment points on suction side do not exist. It is worth noting that the pressure recovery region is also linked to the region of almost constant and high value of the rms wall shear stress. The comparison between LES results shows that the freestream turbulence intensity has a stabilizing effect on wall shear stress as the rms value is smaller. Nevertheless, this intensity does not modify significantly the flow topology parameters. Regarding the rms value of wall shear stress predicted by URANS simulation, one can notice that the level is heavily lower than rms value of LES. The URANS seems to be able to capture some fluctuations but as these fluctuations concerned the Reynolds averaged field, they do not play a part in the transition process which is only modeled using the $\gamma-\operatorname{Re}_{\theta \mathrm{T}}$ model of Menter et al. [13][14].

Figure $7 \mathrm{~b}$ and Figure $7 \mathrm{~d}$ show the time-averaged wall shear stress and its rms value along the blade suction side for $\operatorname{Re}_{2 \text { is }}=140000$. With the same argument as $\operatorname{Re}_{2 \text { is }}=80000$, one can deduce the separation, transition and reattachment points. The agreement between experimental and numerical data is better than the $\mathrm{Re}_{2 \mathrm{is}}=80000$ case. The separation point predicted by RANS and LES simulations is slightly upstream of the experimental one: $\mathrm{x} / \mathrm{C}_{\mathrm{ax}}=$ 0.73 (RANS, LES) and $\mathrm{x} / \mathrm{C}_{\mathrm{ax}}=0.74$ (experimental data). As for the previous Reynolds number $\left(\operatorname{Re}_{2 \text { is }}=80000\right)$, the separation point is located where the isentropic Mach number slope starts to reduce and tends towards a constant value (Figure $5 \mathrm{c}$ and Figure $5 \mathrm{~d}$ ). The Reynolds number has no influence on the predicted transition point as the position is not modified $\left(\mathrm{x} / \mathrm{C}_{\mathrm{ax}}=0.8\right.$ for the steady and unsteady RANS simulations and $\mathrm{x} / \mathrm{C}_{\mathrm{ax}}=0.85$ for LES computation) while the experimental transition point is more downstream with the Reynolds number $\operatorname{Re}_{2 i s}=140000\left(\mathrm{x} / \mathrm{C}_{\mathrm{ax}}=0.77\right.$ vs. $\left.\mathrm{x} / \mathrm{C}_{\mathrm{ax}}=0.74\right)$. As shown in section 5.3, the open bubble is not predicted by CFD. Thus the CFD is unable to capture the modification of the transition point as shown by Babajee and Arts [50] of which results are consistent with the present CFD results. The main discrepancy between the numerical predictions and experimental data concerns the reattachment point. This one is too early for steady and unsteady RANS simulations $\left(\mathrm{x} / \mathrm{C}_{\mathrm{ax}}=0.90\right)$ and slightly downstream for $\operatorname{LES}\left(\mathrm{x} / \mathrm{C}_{\mathrm{ax}}=0.95\right)$ in comparison to experimental data $\left(\mathrm{x} / \mathrm{C}_{\mathrm{ax}}=0.938\right)$. This discrepancy in location of reattachment point is visible in Figure $5 \mathrm{c}$ and Figure $5 \mathrm{~d}$ and mainly results from the bursting prediction as the bubble is short and long, respectively for RANS and LES computations. 


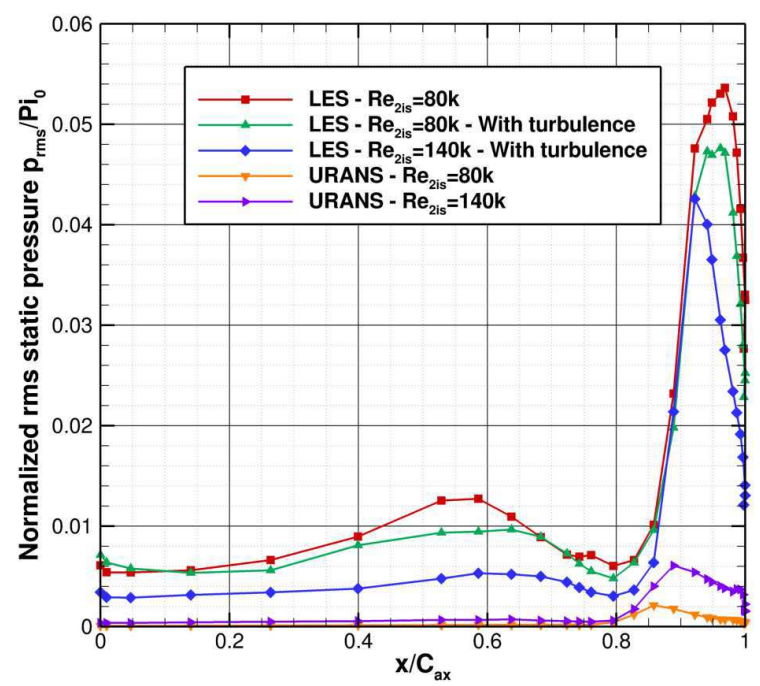

(a) Suction side

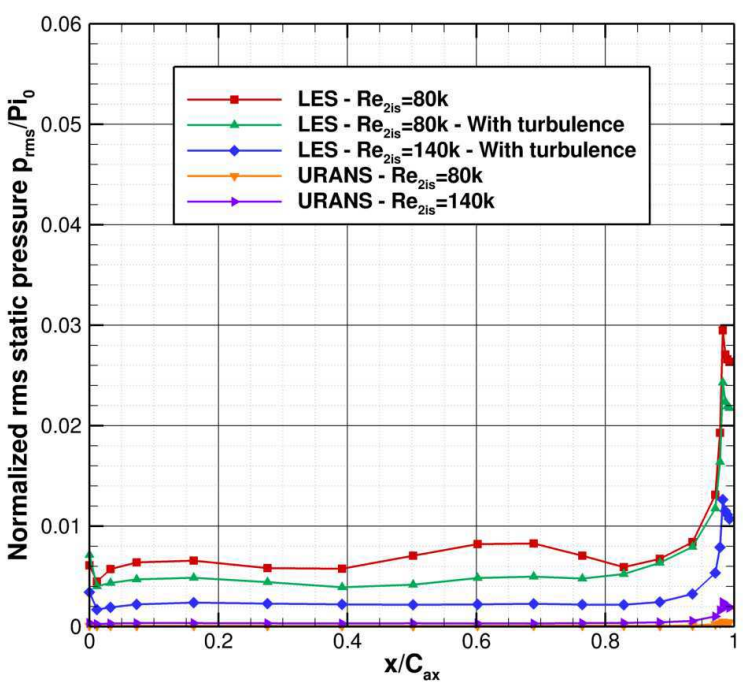

(b) Pressure side

Figure 8: Distribution of rms static pressure on T106C blade at midspan

Several numerical probes have been put on the blade wall. The rms static pressure extracted from these probes is plotted in Figure 8. With respect to the time-averaged isentropic Mach number (Figure 5), the rms static pressure increases near the velocity peak on the suction side (Figure 8a), especially for the lowest Reynolds number without freestream turbulence. Then the rms level decreases up to the normalized abscissa 0.8. The point is located within the separation bubble of which the laminar nature is confirmed. From the normalized abscissa 0.85 , the rms static pressure strongly increases. This is due to the laminar-turbulence transition within the bubble and to the pressure recovery. This normalized abscissa is consistent with the transition point determined using the rms value of wall shear stress. The rms level of the case $\operatorname{Re}_{2 \text { is }}=140000$ decreases first near the trailing edge because the transition is completed more quickly. The laminar separation bubble is shorter for this Reynolds number than for the lowest Reynolds number $\left(\operatorname{Re}_{2 \mathrm{is}}=80000\right)$. At the trailing edge, the rms pressure is non-zero because of the vortex shedding. Compared to LES, the rms of URANS simulation is eight times smaller. The unsteadiness predicted by URANS simulation has clearly small amplitude. This result will be discussed later. The transition point is observed at $\mathrm{x} / \mathrm{C}_{\mathrm{ax}}=0.80$ as previously shown by the rms value of wall shear stress.

The freestream turbulence influences only the rms level, not the rms shape. The level is smaller with freestream turbulence because of the stabilizing effect of the incoming turbulence via the receptivity phenomenon. Due to the freestream turbulence, the laminar boundary layer contains small perturbations without becoming turbulent. These perturbations have a stabilizing effect on the boundary layer. It explains that the separation point predicted with freestream turbulence is downstream the separation point without turbulence (Figure $5 b$ ). As the freestream turbulence intensity is small and the predicted laminar separation bubble is long, there is no difference on the rms shape.

On the pressure side, the rms levels are small except near the trailing edge. In the vicinity of the trailing edge, the laminar boundary layer becomes turbulent as shown by the turbulent kinetic energy in Figure 9d and Figure 11d. The transition is combined with the vortex shedding. They induce static pressure fluctuations near the trailing edge. It should be noticed that the boundary layer remains laminar up to the trailing edge because of the damping of instability waves (e.g. Tollmien-Schlichting waves) due to the favorable pressure gradient.

\subsection{Steady and time-averaged flows}




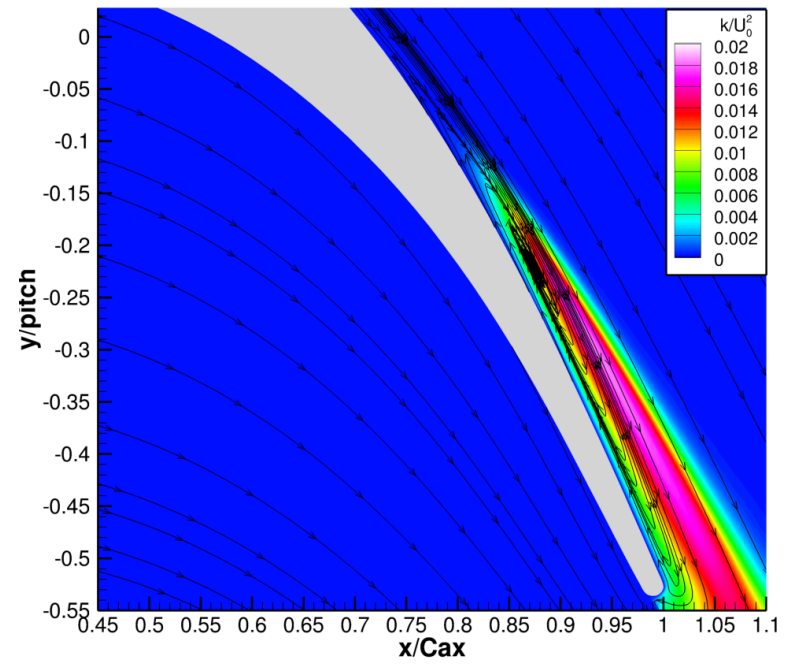

(a) Coarse Mesh - RANS - Transition model

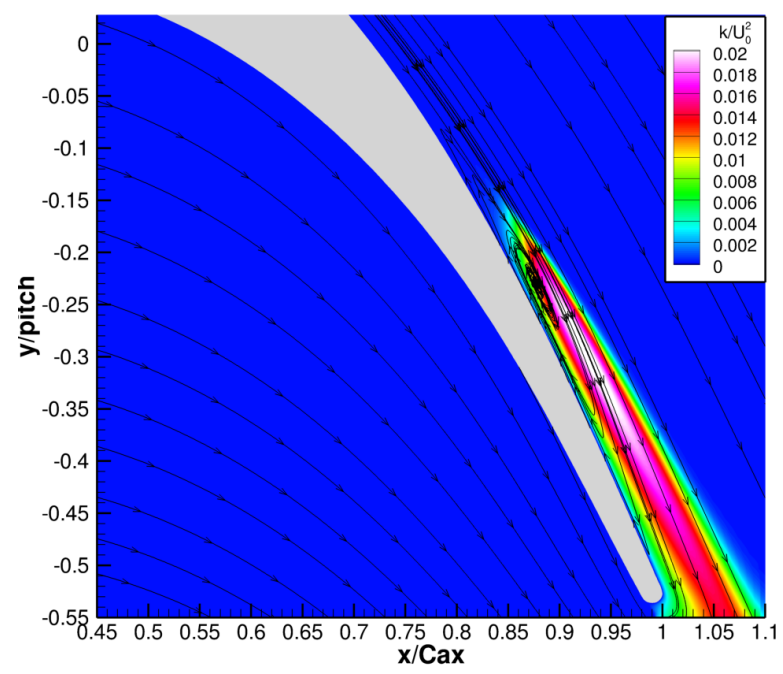

(c) Fine Mesh - RANS - Transition model

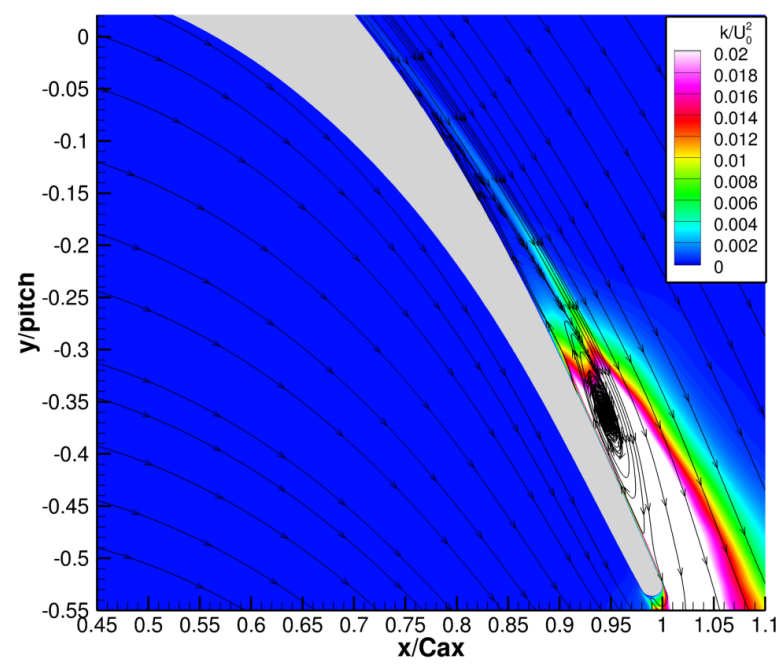

(e) Fine Mesh - Time-averaged LES

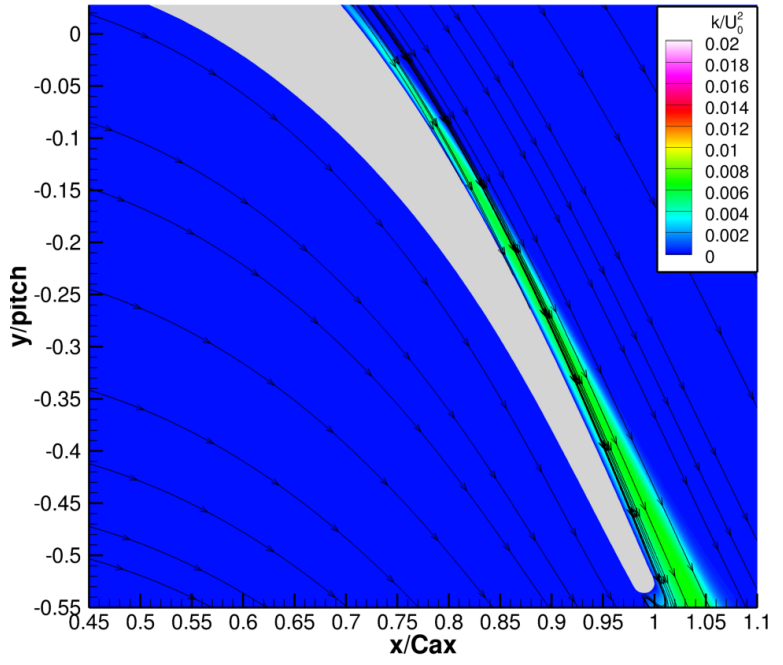

(b) Fine Mesh - RANS - Fully turbulent

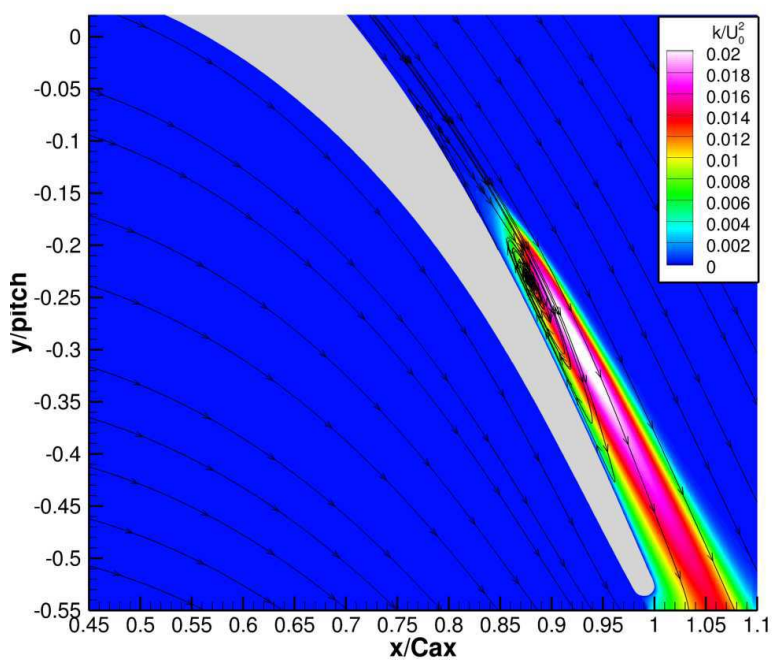

(d) Fine Mesh - Time-averaged URANS - Transition model

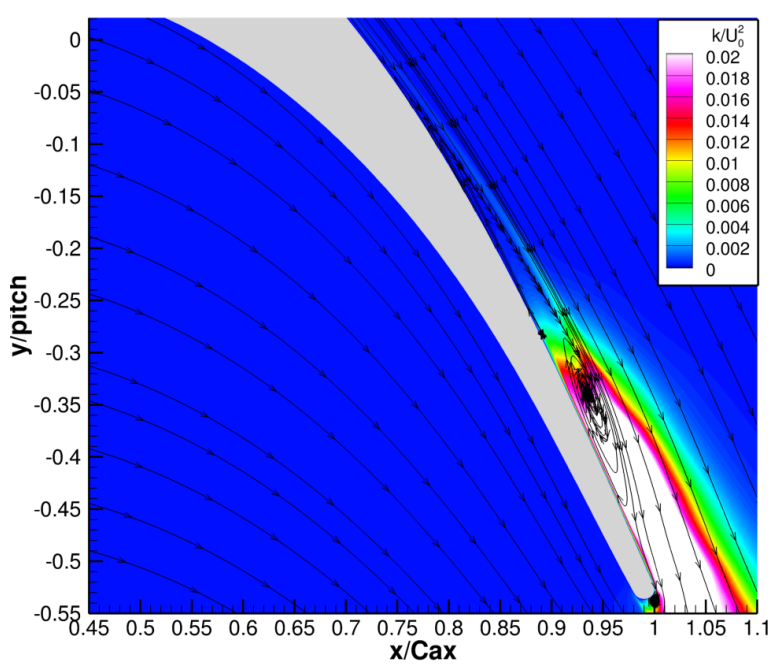

(f) Fine Mesh - Time-averaged LES Random generated turbulence

Figure 9: Turbulent kinetic energy for $\mathrm{Re}_{2 \text { is }}=80000$ 
Figure 9 depicts the turbulent kinetic energy distribution on the rear part of the blade at midspan for $\operatorname{Re}_{2 \text { is }}=80000$ while Figure 10 shows the evolution of wall distance $\eta$ where the velocity is null and the maximum of turbulent kinetic energy in the boundary layer and laminar separation bubble along the blade suction side for the same Reynolds number. The fully turbulent simulation is unable to capture the laminar separation bubble (Figure 9b). As the turbulent kinetic energy is not equal to zero before the potential separation point, the boundary layer does not separate and remains attached all along the suction side. In the absence of separation, the turbulent kinetic energy level is lower than the level predicted by the other simulations due to lower production.

The comparison between the steady and unsteady RANS simulations with the transition model and the LES simulation shows a significant difference in laminar separation bubble prediction (Figure 9c, Figure 9d and Figure 9e) while there is no major discrepancy between the steady and the time-averaged unsteady RANS predictions. The bubble obtained with RANS simulation is thinner and shorter than with LES. The transition point can be estimated from the turbulent kinetic energy distributions (Figure 10). The transition point predicted with LES is downstream of the point obtained with RANS simulation $\left(x / C_{a x}=0.80\right.$ for RANS simulations and $\mathrm{x} / \mathrm{C}_{\mathrm{ax}}=0.85$ for LES) while the separation points are closer $\left(\mathrm{x} / \mathrm{C}_{\mathrm{ax}}=0.71\right.$ for RANS simulations, $0.72 \mathrm{C}_{\mathrm{ax}}$ for LES w/o freestream turbulence and $0.73 \mathrm{C}_{\mathrm{ax}}$ with freestream turbulence). It should be noticed that the separation point location is predicted downstream of the experimental point $\left(\mathrm{x} / \mathrm{C}_{\mathrm{ax}}=0.64\right)$. The separation point location determined using the streamlines is in good agreement with the location defined from the wall shear stress (Figure 7a) and the wall distance relative to zero velocity (Figure 10). The transition point is linked to the significant turbulent kinetic energy rise. Although URANS simulations capture unsteadiness, the transition is only triggered when the Reynolds number based on momentum thickness reaches the computed critical Reynolds number. The discrepancy in bubble prediction between the two simulations leads to an earlier pressure recovery for the RANS simulation. Moreover, the RANS simulation with transition model and LES simulation predict only a large bubble, not an open bubble as observed in the experiment [34].

Nevertheless, the open bubble is predicted with the coarse mesh (Figure 9a). For the coarse mesh, the separation point obtained with the RANS simulation based on the transition model is located upstream of $0.7 \mathrm{C}_{\mathrm{ax}}$ i.e. at $0.69 \mathrm{C}_{\mathrm{ax}}$ while this point is located at $0.71 \mathrm{C}_{\mathrm{ax}}$ for all other RANS computations with the transition model (Figure 10). This separation point discrepancy explains partially the difference in open bubble prediction with the three meshes. Although the production of turbulent kinetic energy is located upstream for the coarse mesh, the flow is still separated at the trailing edge. As the bubble is thicker with the coarse mesh, the production of turbulent kinetic energy is smaller than the fine mesh. This higher production on fine mesh leads to the rapid transition and reattachment leading to a long bubble. Thus, the more upstream the separation point is, the more downstream the reattachment point is and longer the bubble is. It could explain that an error in separation point location leads to discrepancies in transition and reattachment points. The mesh density has a strong influence on the predictions of the boundary layer and of the laminar separation bubble, especially the mesh quality in the vicinity of the wake as shown by Babajee and Arts [50]. The maximum of streamwise, wall-normal and spanwise distances in wall unit $\left(\mathrm{x}^{+} / \mathrm{y}^{+} / \mathrm{z}^{+}\right)$ are respectively $1300 / 1.3 / 1500$ and $22 / 0.14 / 180$ for the coarse and fine meshes of RANS simulations. The pressure and suction sides are discretized by 233 and 898 points for the coarse and fine meshes, respectively. Thus, the transition model of Menter et al. [13][14] is clearly mesh-dependent. In the present study, only three meshes are used for RANS simulations. Thus the effect of $\mathrm{x}^{+}, \mathrm{y}^{+}$and $\mathrm{z}^{+}$distributions cannot be investigated separately and no mesh criterion can be defined for the simulation of laminar separation bubble using RANS computation with transition model. As previously shown, the comparison between medium and fine mesh shows that the fine mesh is close to the mesh convergence limit for 
RANS simulations (Figure 5a). Finally, although this result was not unexpected, the mesh should be generated carefully. The present study does not suggest running on coarse grid as a modeling error can be canceled by a mesh error and leads to a false conclusion.

The comparison of LES results (Figure 9e and Figure 9f) highlights the effect of freestream turbulence. The separation and transition points obtained with the large-eddy simulation with freestream turbulence are located slightly downstream of the simulation without turbulence. The laminar separation bubble thickness and length are smaller with freestream turbulence due to the influence of incoming turbulence on the boundary layer development. As the freestream turbulence intensity is very small $(0.9 \%)$, the effect of turbulence is not very significant. For higher intensities, the effect should be more noticeable and the transition process should be modified [27]. McAuliffe and Yaras [55] show that streamwise streaks appear in the boundary layer at high freestream turbulence level at separation point $(1.45 \%)$. The freestream turbulence effect is smaller than the turbulence modeling/resolving method impact. With LES, the bubble is thicker and turbulent kinetic energy is produced far downstream than URANS computation. The maximum of turbulent kinetic energy is closer to the trailing edge for LES leading to a rapid decrease of bubble size as flow reattaches at 0.99 $\mathrm{C}_{\mathrm{ax}}$.

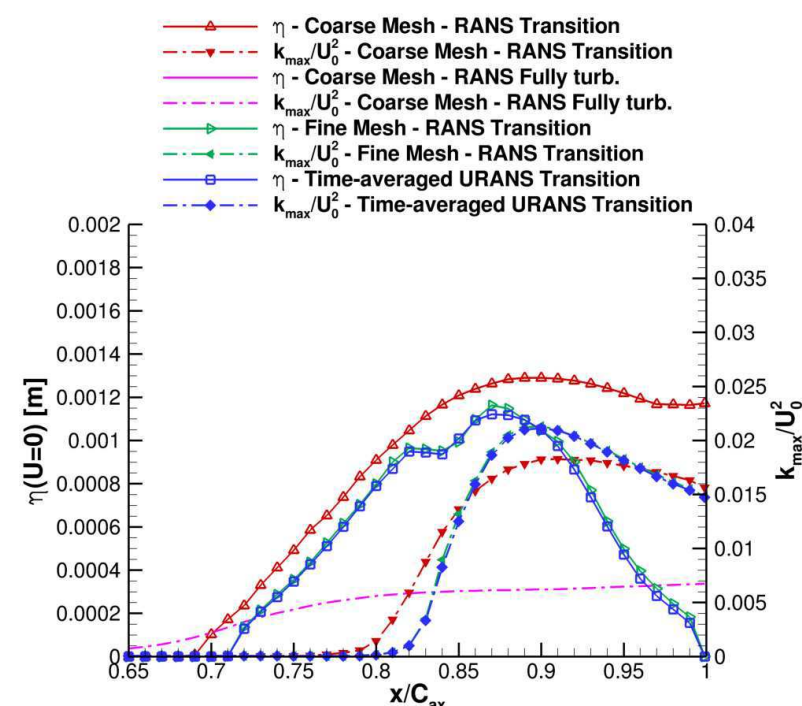

(a) RANS and time-averaged URANS

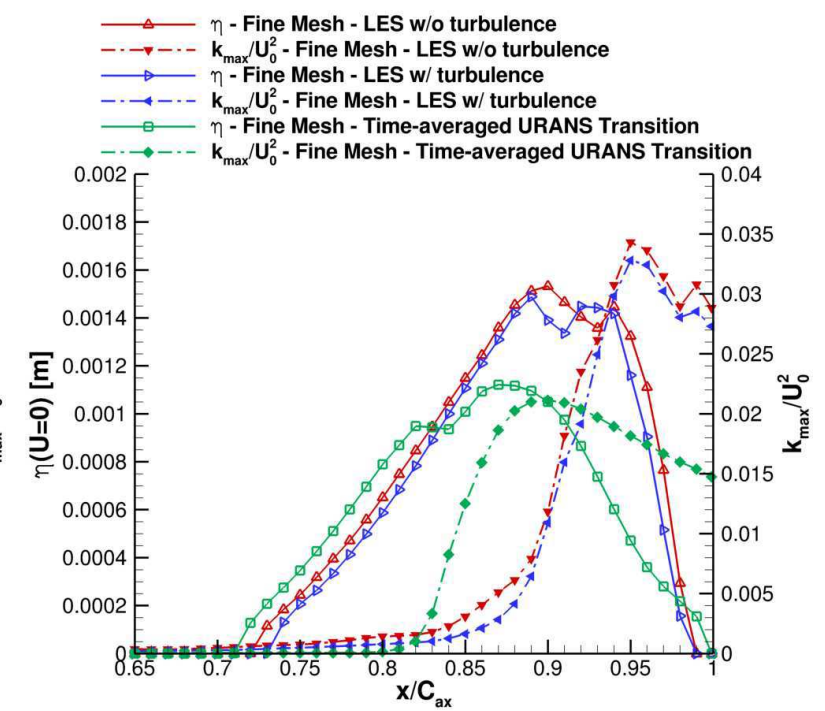

(b) Time-averaged URANS and LES w/o and $\mathrm{w} /$ freestream turbulence

Figure 10: Wall distance $\eta$ where $U=0$ and turbulent kinetic energy maximum normalized by freestream velocity at $\operatorname{Re}_{2 \text { is }}=80000$ 


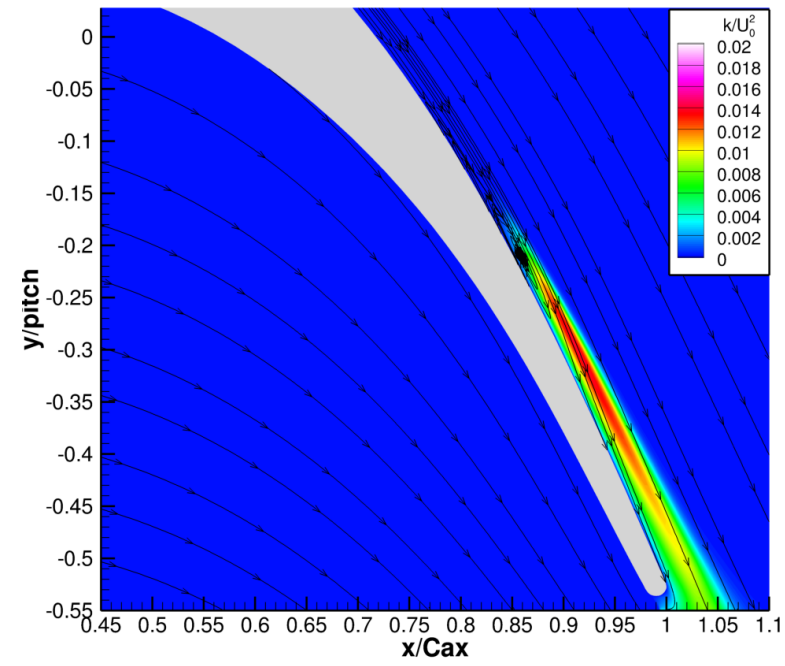

(a) Coarse Mesh - RANS - Transition model

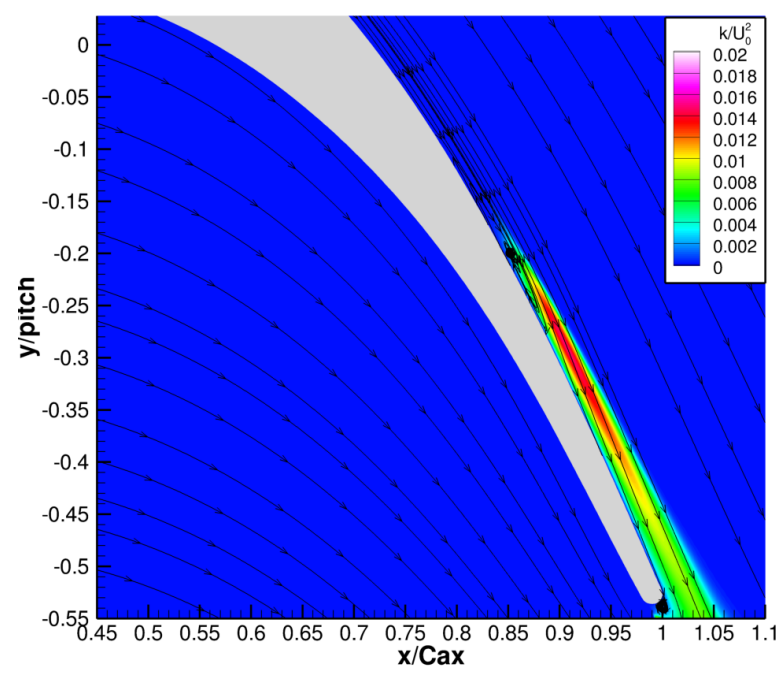

(c) Fine Mesh - RANS - Transition model

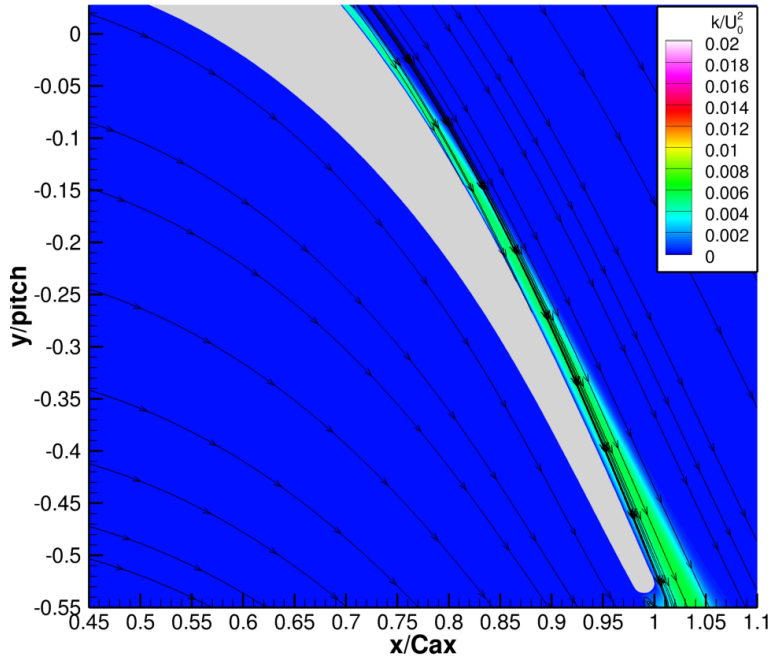

(b) Fine Mesh - RANS - Fully turbulent

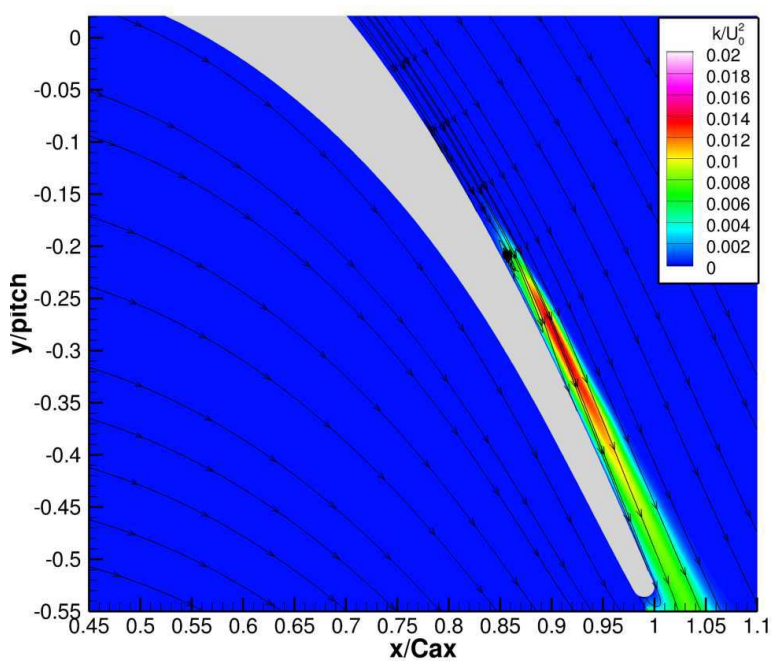

(d) Fine Mesh - Time-averaged URANS - Transition model

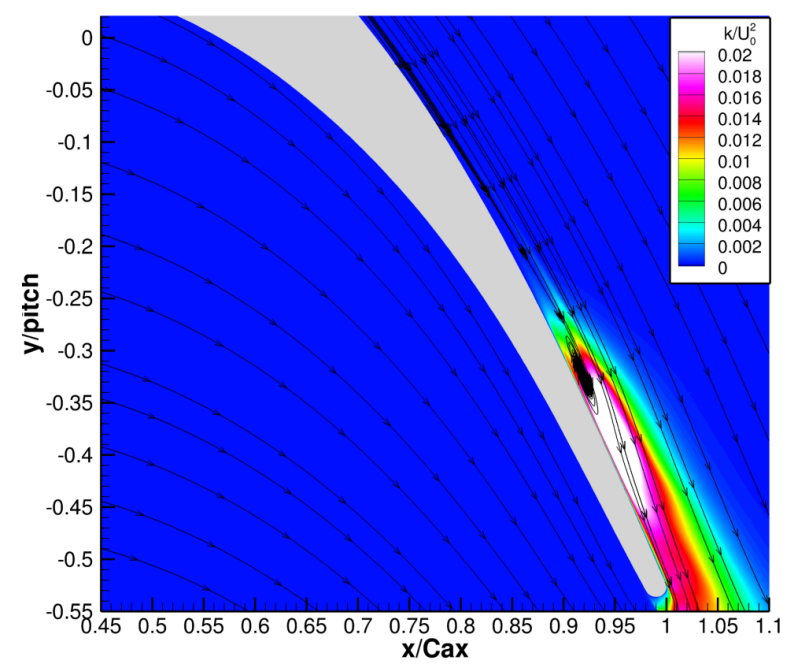

(e) Fine Mesh - Time-averaged LES - Random generated turbulence

Figure 11: Turbulent kinetic energy for $\operatorname{Re}_{2 \text { is }}=140000$ 
The turbulent kinetic energy distributions on the rear part of the blade at midspan for $\operatorname{Re}_{2 \text { is }}=$ 140000 are depicted in Figure 11. Figure 12 shows the evolution of the wall distance $\eta$ and the maximum of turbulent kinetic energy for Re2is $=140000$. For all computations, the bubble is not open as in experiments. Contrary to the lowest Reynolds number, the effect of mesh density is weak: the prediction of a small laminar separation bubble is quite similar for the RANS simulations with transition modeling (Figure 11a and Figure 11c). As for the previous studied Reynolds number, the fully turbulent simulation is unable to capture the laminar separation bubble. It is worth noting that the steady and time-averaged unsteady RANS predictions are quite identical.

The LES simulation predicts a long laminar separation bubble while the RANS simulation captures a small one. This discrepancy is explained by the location of the separation, transition and reattachment points (more details are given in section 5.2). The separation point of LES simulation is located upstream of the separation point of RANS simulation $\left(0.74 \mathrm{C}_{\mathrm{ax}}\right.$ vs. $0.75 \mathrm{C}_{\mathrm{ax}}$ ). On the contrary, the transition point obtained with LES is downstream of the point predicted with RANS turbulence model $\left(0.85 \mathrm{C}_{\mathrm{ax}}\right.$ vs. $\left.0.80 \mathrm{C}_{\mathrm{ax}}\right)$. The reattachment point is more downstream than URANS, at $0.95 \mathrm{C}_{\mathrm{ax}}\left(0.90 \mathrm{C}_{\mathrm{ax}}\right.$ for URANS). Moreover, the production of turbulent kinetic energy increases downstream for LES despite the location of separation point. This late rise explains the location of reattachment point and the long bubble predicted by LES at $\operatorname{Re}_{2 \text { is }}=140000$ instead of a small bubble for URANS computation. In comparison to $\operatorname{Re}_{2 \text { is }}=80000$, the separation and reattachment points are respectively more downstream and upstream for RANS and URANS computations with transition model than for LES. For all Reynolds number, the bubble is thicker and longer for LES.

Experimentally the bursting is observed for $\mathrm{Re}_{2 \mathrm{is}}=140000$. For higher Reynolds numbers, the bubble is small while for lower Reynolds numbers, it is long. Thus the RANS simulation predicts the limit between the two bubble types for a Reynolds number lower than 140000 while this limit is higher than this Reynolds number for LES simulation. Moreover, the turbulent kinetic energy is higher for LES results. This is due to the bubble type as well as to the coherent structures ejected from the bubble.

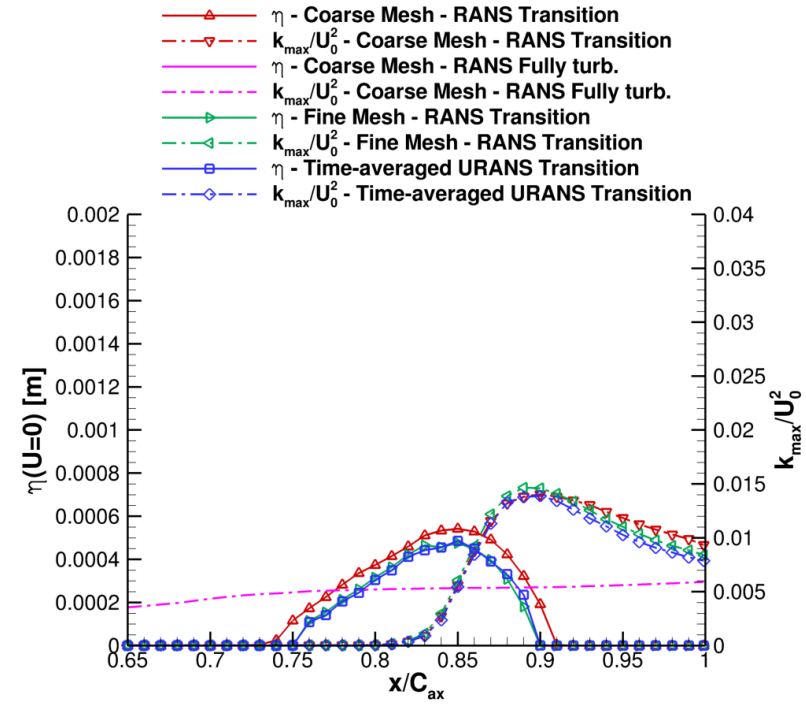

(a) RANS and time-averaged URANS

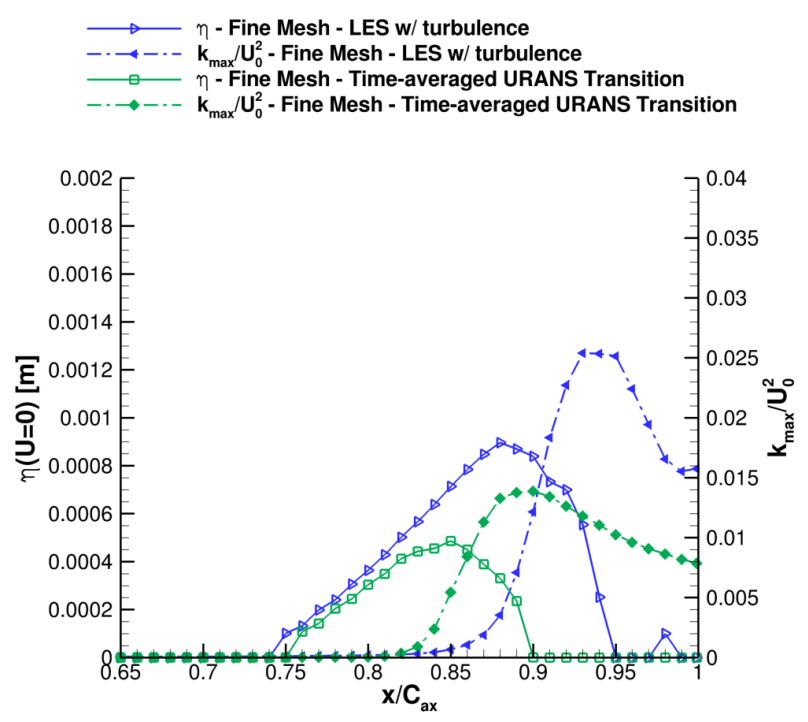

(b) Time-averaged URANS and LES with freestream turbulence

Figure 12: Wall distance $\eta$ where $\mathrm{U}=0$ and turbulent kinetic energy maximum normalized by freestream velocity at $\operatorname{Re}_{2 \mathrm{is}}=140000$ 


\section{Unsteady LES analysis}

\subsection{Unsteady flow}

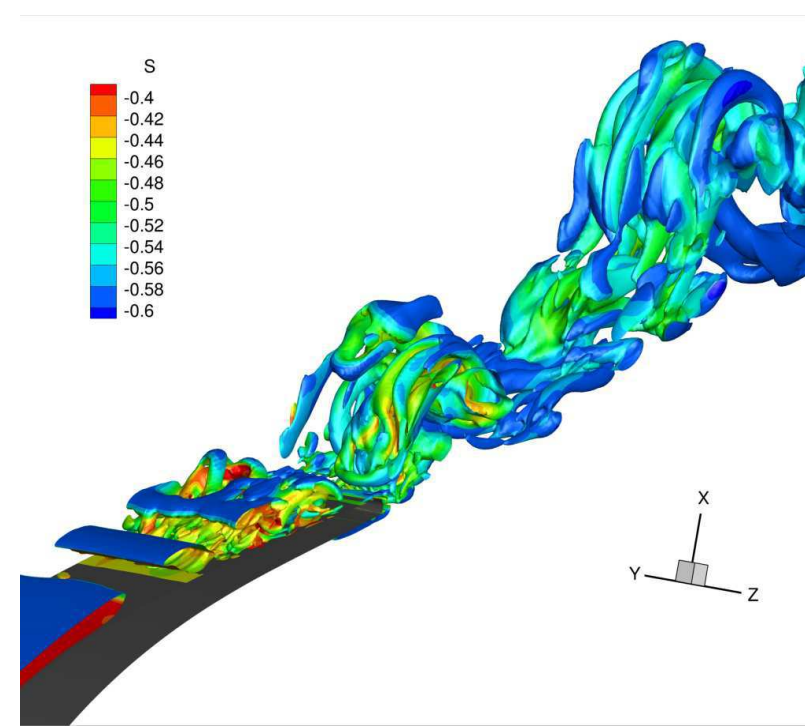

(a) Isosurface of $\mathrm{Q}$ criterion colored by entropy - $\mathrm{QC}_{\mathrm{ax}} / \mathrm{U}_{\infty}{ }^{2}=10$

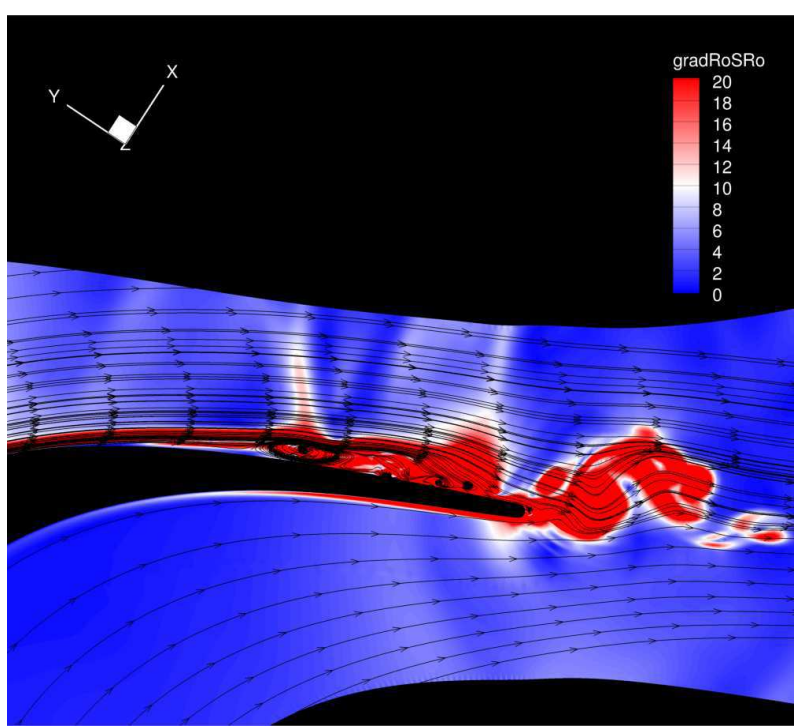

(b) Contours of $\frac{\|\operatorname{grad}(\rho)\|}{\rho}$ (called gradRoSRo in this figure and the followings) and streamlines at midspan in the rear part of the blade

Figure 13: Instantaneous distribution of Q criterion isosurface (a), $\frac{\|\operatorname{grad}(\rho)\|}{\rho}$ contours (b) for $\operatorname{Re}_{2 \text { is }}=80000$ without freestream turbulence

Figure 13a depicts the isosurface of $\mathrm{Q}$ criterion colored by entropy $\left(\mathrm{Q} .\left[\mathrm{C}_{\mathrm{ax}} / \mathrm{U}_{\infty}\right]^{2}=10\right)$ while Figure 13b shows the instantaneous contours of $\frac{\|\operatorname{grad}(\rho)\|}{\rho}$ and streamlines at midspan in the rear part of the blade for the case $\mathrm{Re}_{2 \mathrm{is}}=80000$ without freestream turbulence. A separated shear layer is created at the separation point observed at the left of Figure 13b. It develops above the suction side and is subject to flapping. The separated shear layer rolls-up. As shown by Figure 13a, it generates two-dimensional vortices which are distorted in the spanwise direction. It leads to three-dimensional eddies. They are convected downstream and move closer to the wall before breaking into smaller scales. The vortices are ejected from the separated shear layer, are stretched and interact with the wake originating from the pressure and suction side boundary layers. As a result the vortices pair and large structures are observed far from the trailing edge (Figure 14a). This mechanism is nearly independent of the freestream turbulence intensity and of the isentropic exit Reynolds number as long as the predicted laminar separation bubble is long. As the freestream intensity is weak, there is no significant effect on the ejected structures as shown by the comparison between Figure 14a and Figure 14b. As previously shown, for the isentropic exit Reynolds number 140 000, the LES computation predicts a long bubble while the RANS simulation a small bubble. The predicted flows by LES at the two isentropic exit Reynolds numbers 80000 and 140000 differ only from the locations of separation, transition and reattachment points and from the size of the bubble and of coherent structures ejected. Nevertheless the involved mechanism seems to be similar between these two simulations. As a shear layer is created, instability occurs. It is not the Tollmien-Schlichting instability which already exists but the Kelvin- 
Helmholtz instability. The mechanism previously described is typical of transition driven by the Kelvin-Helmholtz instability and was also observed by Watmuff [56], Yang and Voke [57], Roberts and Yaras [58] and Richez et al. [19]. In order to prove the role of the KelvinHelmholtz instability in transition phenomenon, the criterion of Chandrasekhar [59] is used: $0<k h<C$ where $k$ and $h$ are respectively the instability wave number and the characteristic length of the shear layer. The constant $C$ is the upper limit of unstable region of KelvinHelmholtz instability. It is 1.2785 for a shear layer with a linear velocity profile and 1 for a hyperbolic tangent profile. In the present study, $h$ is evaluated at the separation point and $k$ is computed from the wavelength $\lambda(k=2 \pi / \lambda)$ which is evaluated as the instantaneous distance between two vortices with the same sign of spanwise vorticity component. The results are given in Table 2. All cases fulfill the previous criteria, thus confirming the role of the KelvinHelmholtz instability.

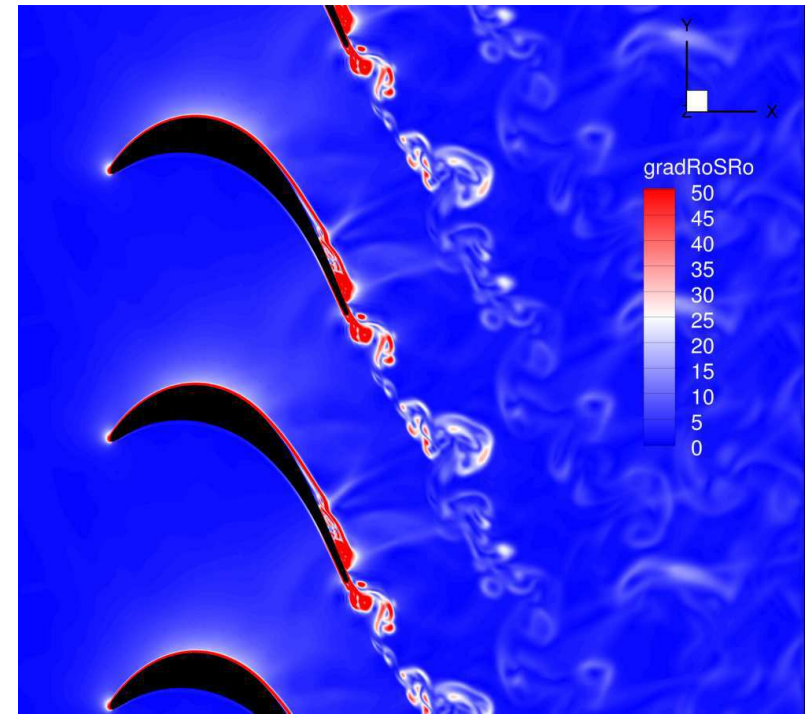

(a) $\mathrm{Re}_{2 \mathrm{is}}=80000$ without freestream turbulence

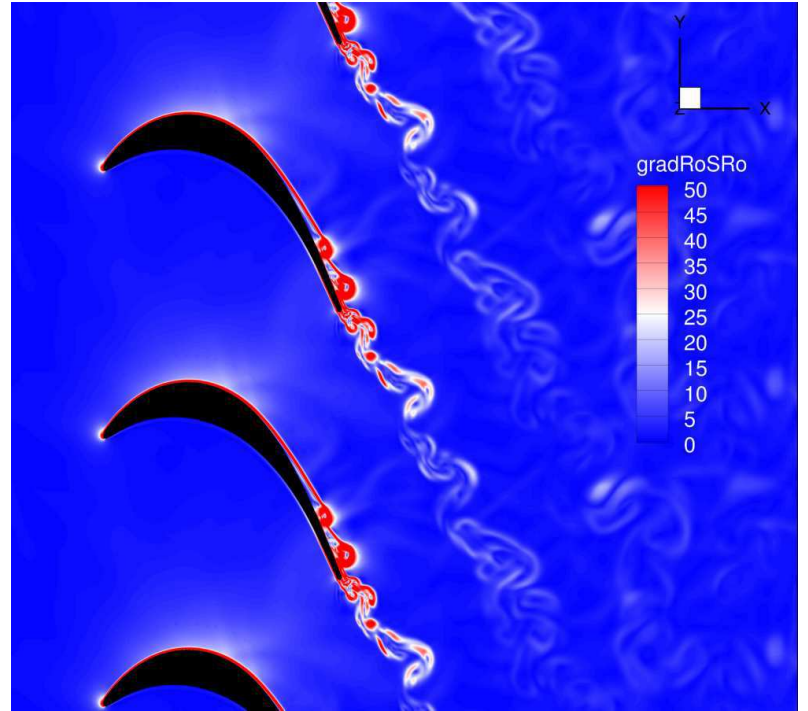

(b) $\mathrm{Re}_{2 \mathrm{is}}=80000$ with freestream turbulence

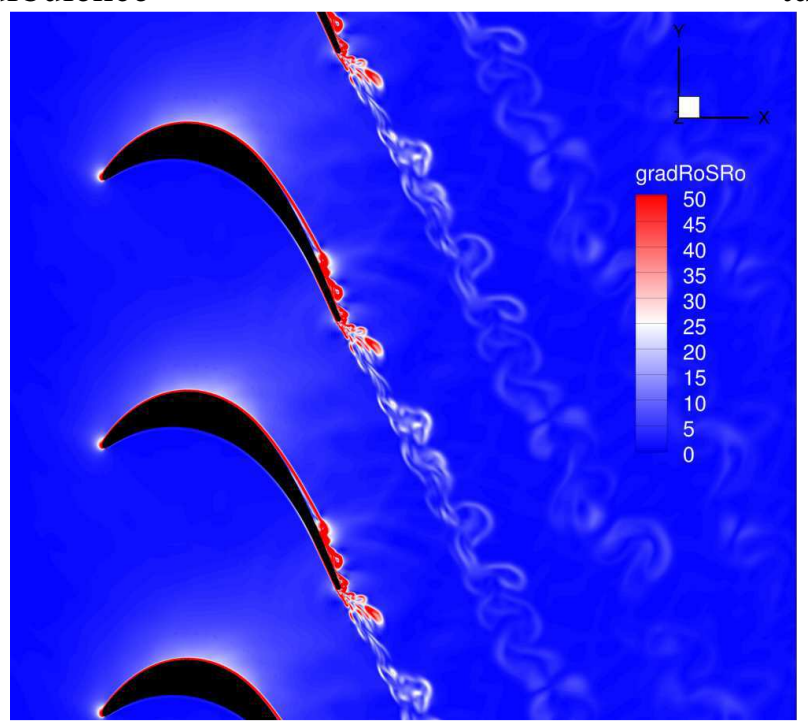

(c) $\mathrm{Re}_{2 \mathrm{is}}=140000$ with freestream turbulence

Figure 14: Contours of $\frac{\|\operatorname{grad}(\rho)\|}{\rho}$ at midspan for several blade passages obtained by

translation 
Table 2: Criteria for Kelvin-Helmholtz instability

\begin{tabular}{|l|c|c|c|}
\hline Isentropic exit Reynolds number & 80000 & 80000 & 140000 \\
\hline Freestream turbulence & No & Yes & Yes \\
\hline$k h$ & 0.9132 & 0.653 & 0.7409 \\
\hline
\end{tabular}

Figure 13Figure 14 shows that wave reflection occurs at the outflow boundary condition due to the static pressure condition and insufficient mesh cell stretching near the outflow boundary condition, especially at the lowest Reynolds number and without freestream turbulence. Due to the large distance between the trailing edge and this boundary condition one and a half chord - the reflected wave is damped far from the trailing edge and does not influence the laminar-turbulent transition mechanism prediction. Moreover, an acoustic wave is created near the trailing edge, over the laminar separation bubble as already found by Raverdy et al. [20].

\subsection{Spectral analysis}

Power spectral densities are a wealth of information about the unsteadiness of the flow. This spectral analysis aims at determining the frequencies and the spatial correlations of the flow structures, especially vortex shedding of the separation area, Kelvin-Helmholtz instabilities of the shear layer and wake vortices. Figure 15a shows the probe locations where static pressure and spanwise velocity are extracted from the simulation along ten periods $T=\mathrm{C}_{a x} / \mathrm{U}_{\infty}$. These probes are specially selected according to the flow topology as depicted by the contours of $\frac{\|\operatorname{grad}(\rho)\|}{\rho}$. The PSD are based on the Welch method [60] using an overlap of $50 \%$ and ten Hann windows with a linear mean for each. The frequency resolution is $1000 \mathrm{~Hz}$. For lower frequencies, the signals must be longer and the cost of the simulation would be too expensive: for a first frequency at $100 \mathrm{~Hz}$, the signal must be ten times longer. It is possible to estimate the LES cut-off frequency for all cases according to the method of Boudet et al. [17]. This estimation is based on the cubic-root of the cell volume and the velocity fluctuations. The LES cut-off frequency is close to $300 \mathrm{kHz}$.

The PSD function of static pressure fluctuations $G_{p}(f)$ has been plotted in log-log scale in Figure $15 b$ and Figure 15c, respectively for the LES without freestream turbulence and the URANS simulation at $\mathrm{Re}_{2 \mathrm{is}}=80000$. For LES results, from the first probe located at the leading edge, the levels increase until the first probe in the wake (probe 7). Then the levels decrease due to the interaction between the flow structures and the diffusion and dissipation of these structures. From the third probe, levels of PSD are strongly higher for the frequencies close to $100 \mathrm{kHz}-300 \mathrm{kHz}$. As the estimated cutoff frequency is close to $300 \mathrm{kHz}$, the power spectral density drops from this frequency. A finer mesh is required to resolve smaller turbulent scales. In comparison to LES results, the energy is concentrated in lower frequencies in URANS. The fluctuations of small scales are dissipated by URANS modeling, especially after the trailing edge.

Figure $15 \mathrm{~b}$ shows that the $-7 / 3$ slope is observed between 5 and $60 \mathrm{kHz}$ along the suction side from probe 3 which is close to the transition point. Thus, these probes capture the contribution of turbulent-turbulent interaction to the pressure fluctuations. Beyond $60 \mathrm{kHz}$ and up to 200 $\mathrm{kHz}$, probes located close to the trailing edge highlight a $-11 / 3$ slope which is relative to the turbulence - mean shear interaction. So a significant part of the inertial subrange is observed in LES results. All these slopes are not captured by URANS simulation as this method is unable to capture the different interaction with mean and fluctuating flow. 


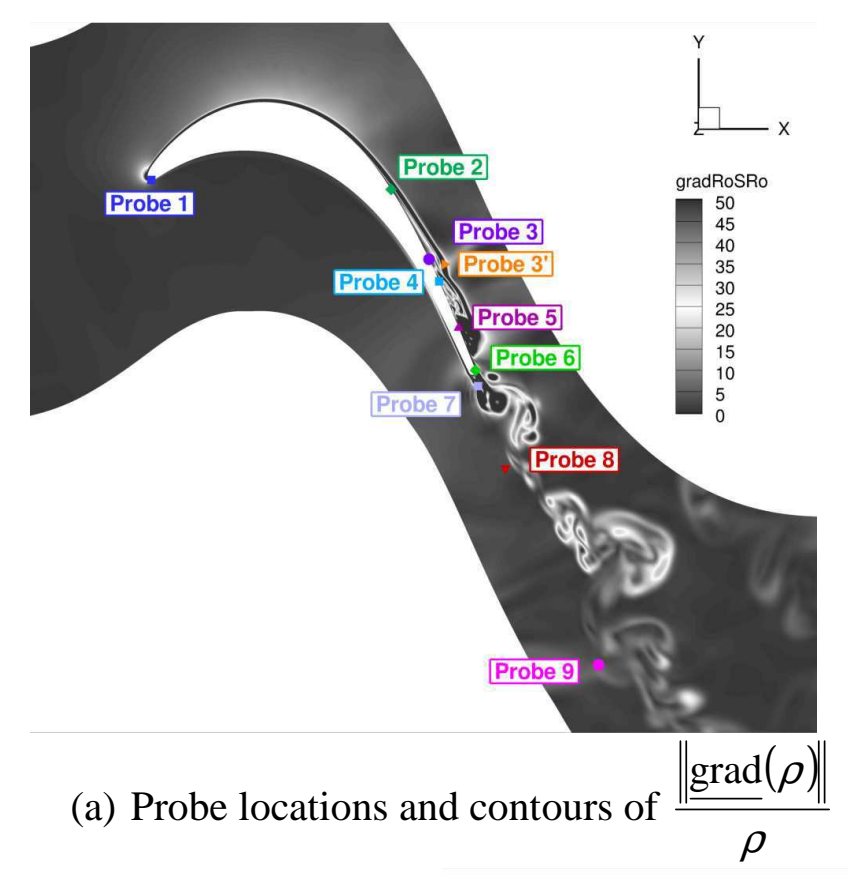

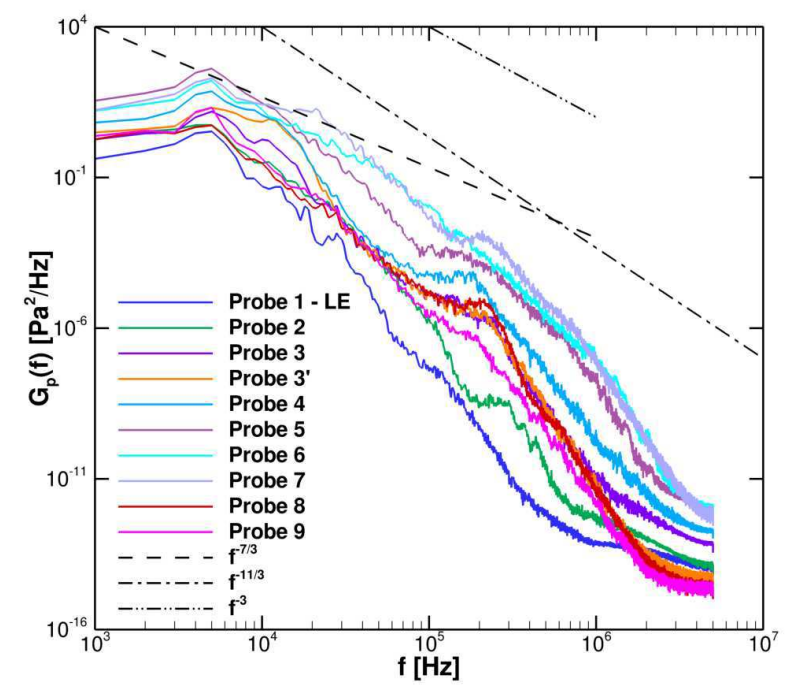

(b) Power spectral density of static pressure - LES w/o freestream turbulence

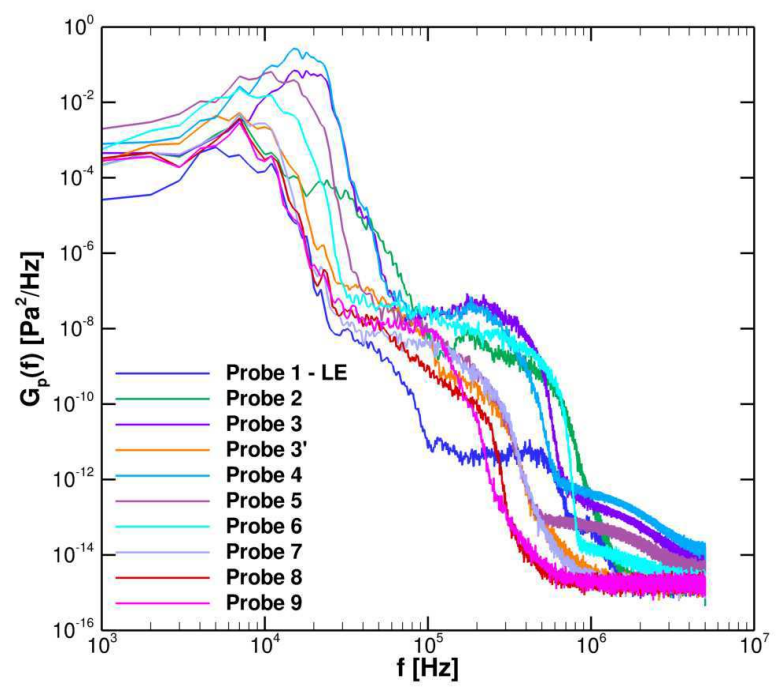

(c) Power spectral density of static pressure - URANS

Figure 15: Power spectral density functions of static pressure (b-c) for several streamwise locations (a) - $\operatorname{Re}_{2 \text { is }}=80000$

Diwan and Ramesh [32] show that the origin of the primary instability in a separation bubble can be traced back to the upstream region of the separation. So the Tollmien-Schlichting (TS) and the Kelvin-Helmholtz $(\mathrm{KH})$ instabilities can be not distinct. They consider that the transition process can be described by KH instability only when the separated shear layer has considerably moved away from the wall. Figure 16 shows the evolution of wall-normal locations of inflectional point of velocity profile and of turbulent kinetic energy maximum. These distances rise from zero after separation point. Along the suction side, the maximum of turbulent kinetic energy is always close to the inflectional point, especially before the transition point. Thus the ratio used by Diwan and Ramesh [32], is close to 1 and shows that the disturbance is due to inflectional mode i.e. $\mathrm{KH}$ instability. It should be noticed that as no stability analysis is performed in the present study, the wall-normal distances relative to most amplified modes cannot be determined and the shift from TS to KH instability cannot be proven using this wall-normal distance ratio. In the rear part of the bubble, discrepancies are 
observed. These ones are due to the turbulence production after the transition point and to the reattachment of flow.

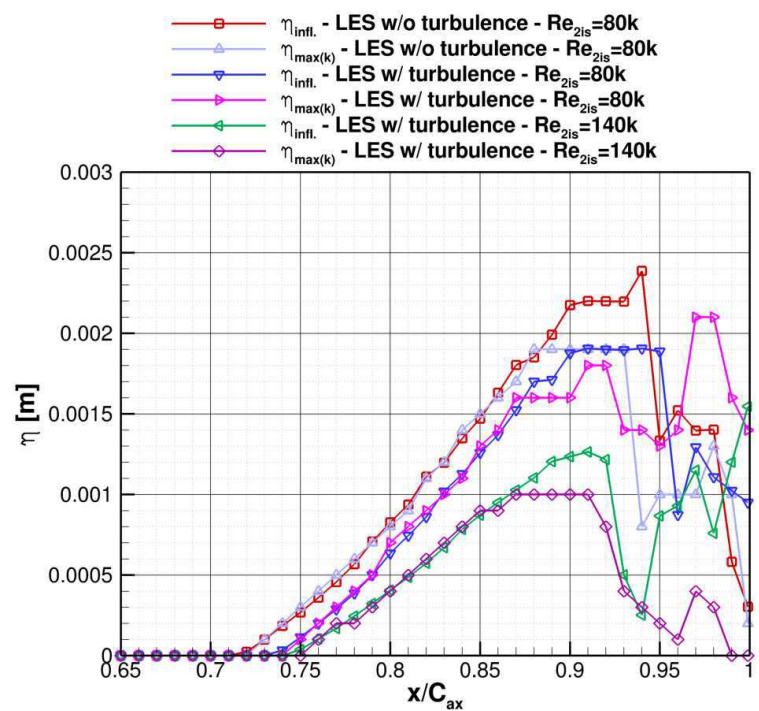

Figure 16: Wall-normal locations of inflectional point and of maximum of turbulent kinetic energy.

McAuliffe and Yaras [33] show that both the TS and KH instabilities can involve in transition process if they occur at the similar frequency (case 1 of the original article, strong adverse pressure gradient). In another case (case 2, milder adverse pressure gradient than case 1), the $\mathrm{KH}$ instability is dominant. In order to distinguish these two instabilities, McAuliffe and Yaras [33] compute the dominant frequency of the growth of TS waves in the preseparated boundary layer using the correlation of Walker [62] (equation (11)) and compare it to the observed dominant frequency.

$$
f_{M A}=\frac{3.2 U_{e}^{2}}{2 \pi \nu \operatorname{Re}_{\delta^{*}}^{3 / 2}}
$$

The computed frequencies are given in Table 3. The predicted frequencies are similar for a given Reynolds number: $4500 \mathrm{~Hz}$ at $\operatorname{Re}_{2 \mathrm{is}}=80000$ and $5375 \mathrm{~Hz}$ at $\operatorname{Re}_{2 \mathrm{is}}=140000$.

Table 3: Dominant frequencies of TS waves at the separation point.

\begin{tabular}{|l|c|}
\hline Case & fMA [Hz] \\
\hline LES w/o turbulence $-\mathrm{Re}_{2 \text { is }}=80000$ & 4500 \\
\hline LES w/ turbulence $-\mathrm{Re}_{2 \text { is }}=80000$ & 4500 \\
\hline LES w/ turbulence $-\mathrm{Re}_{2 \text { is }}=140000$ & 5400 \\
\hline URANS $-\mathrm{Re}_{2 \text { is }}=80000$ & 4500 \\
\hline URANS $-\mathrm{Re}_{2 \text { is }}=140000$ & 5350 \\
\hline
\end{tabular}




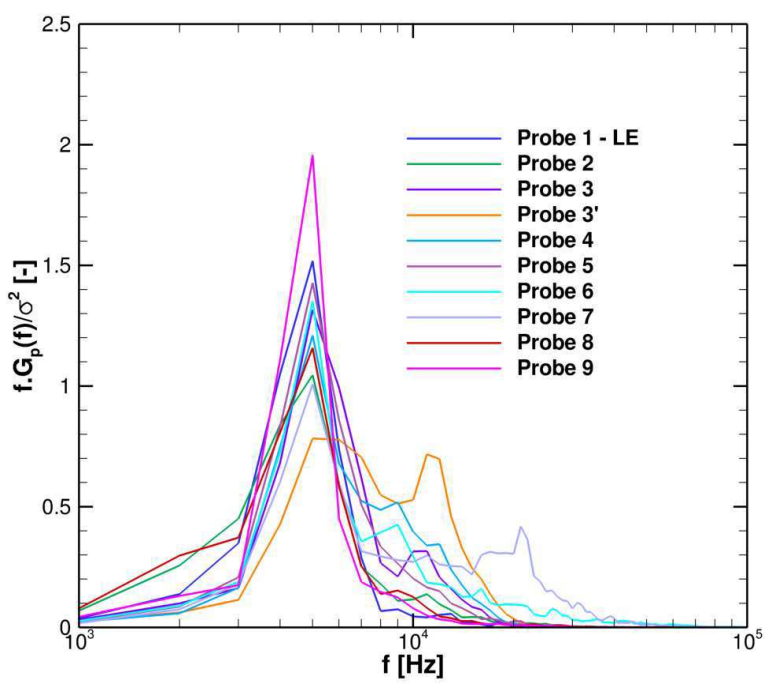

(a) LES w/o freestream turbulence - $\mathrm{Re}_{2 \mathrm{is}}$ $=80000$

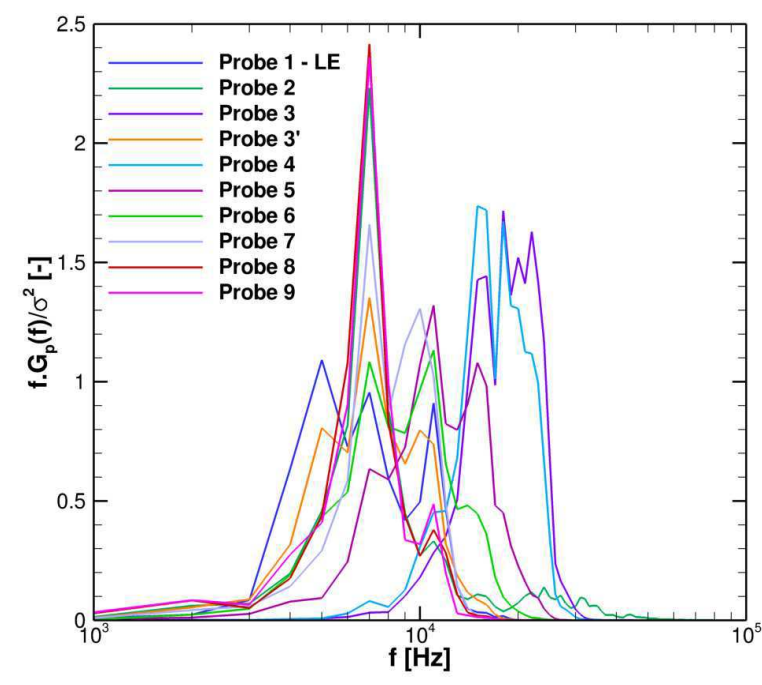

(c) URANS - $\operatorname{Re}_{2 \text { is }}=80000$

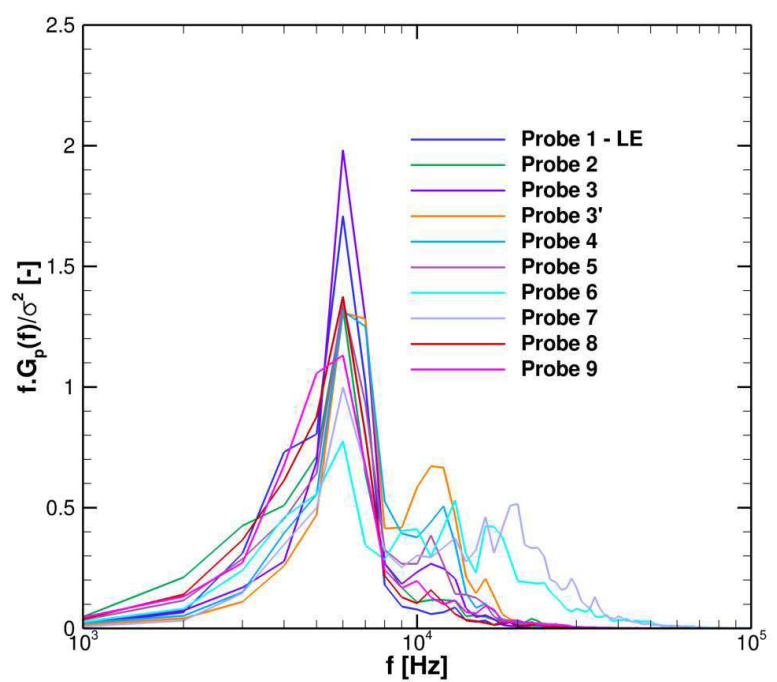

(b) LES w/ freestream turbulence $-\mathrm{Re}_{2 \mathrm{is}}$ $=80000$

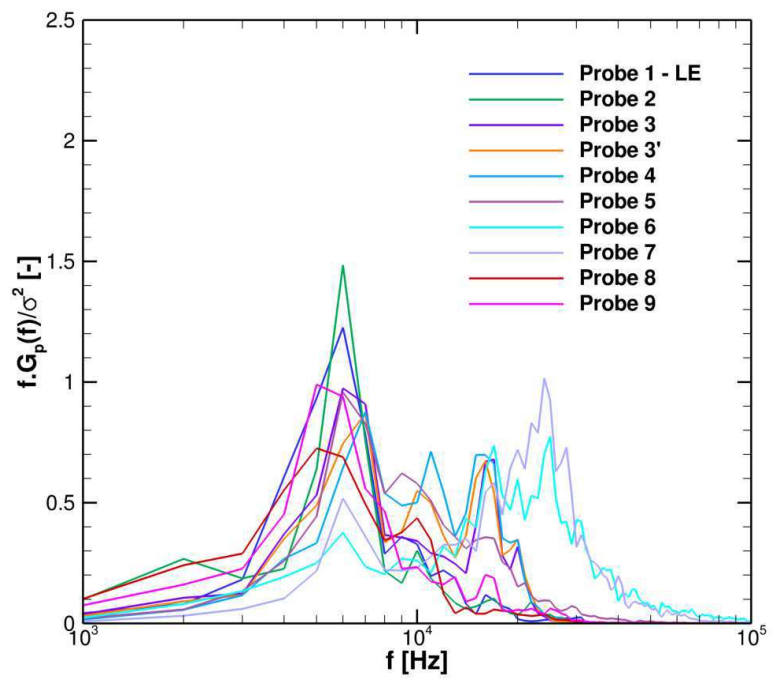

(d) LES w/ freestream turbulence - $\operatorname{Re}_{2 \text { is }}$ $=140000$

Figure 17: Power spectral density functions of static pressure at $\operatorname{Re}_{2 \text { is }}=80000$ and $\operatorname{Re}_{2 \text { is }}=140$ 000 (LES and URANS)

Figure 17 shows the normalized PSD function of static pressure fluctuations and highlights the specific frequencies that drive the separated flow. For LES without freestream turbulence at $\mathrm{Re}_{2 \text { is }}=80000$, a peak is observed at all probes for a frequency of $5 \mathrm{kHz}$, even at the leading edge. This frequency seems to be linked the TS wave i.e. the wall mode of instability [32] and also to the coherent structure ejection and then to the induced pressure wave which propagates upstream (cf. Figure 13b). This frequency is close to the one found by De Saint Victor [63].

The second assumption is confirmed by the observation of this frequency at other probes placed far from the boundary layer as in the wake or in the blade passage. At probe 3', two other peaks are observed. The corresponding frequencies are 6 and $11 \mathrm{kHz}$. Two other probes exhibit a peak at $11 \mathrm{kHz}$ (probes 3 and 4). This is the area where the fluctuating spanwise velocity starts to increase. The peak is relative to the instability of Kelvin-Helmholtz which triggers the laminar-separation transition as shown later. Downstream this area, the amplitude at this frequency decreases. It should be noticed that the $6 \mathrm{kHz}$ frequency is only observed in the vicinity of probe 3'. It may be tied to the flapping of the separated shear layer. At probe 7, 
a peak is observed for the frequency equal to $22 \mathrm{kHz}$. The Strouhal number based on this frequency, the velocity outside the wake and the thickness of the wake near the trailing edge is equal to 0.20 . Thus this frequency is linked to the vortex shedding at the trailing edge. Due to the interaction between vortices, this frequency is not observed further downstream.

The normalized PSD function of the spanwise velocity fluctuations relative to the probes located within the wake is plotted in Figure 18. In LES without freestream turbulence at $\operatorname{Re}_{2 \text { is }}$ $=80000$ (Figure 18a), close to the trailing edge (probe 7), a high number of scales are observed as shown by the vast number of peaks from 5 to $30 \mathrm{kHz}$. These scales do not exist far from the trailing edge where only two peaks are visible. These peaks are relative to larger scales as the frequencies are smaller i.e. large vortices as shown in Figure 14. These vortices are originated from the interaction between the vortices ejected from the laminar separation bubble and the vortices shed at the trailing edge.

In experiment, only one frequency and its higher harmonics are observed: $170 \mathrm{~Hz}$. These frequencies cannot be captured in the present study as the resolution frequency is $1 \mathrm{kHz}$. Moreover, the signal is low-pass filtered at $12 \mathrm{kHz}$. So the frequencies of numerical results cannot be compared to experiment. A ten times longer signal is required but the computational cost would be very expensive.

The freestream turbulence induces a slight modification of the normalized PSD function of static pressure fluctuations (Figure 17a and Figure 17b). The main discrepancy is the frequency relative to the peak of the highest amplitude. With freestream turbulence, this frequency is equal to $6 \mathrm{kHz}$ instead of $5 \mathrm{kHz}$. It is due to the frequency resolution which is 1 $\mathrm{kHz}$. All features previously shown are still available but with a slightly different frequency. Due to the frequency resolution, the peaks previously observed at 5 and $6 \mathrm{kHz}$ are merged in one frequency $(6 \mathrm{kHz})$. Another discrepancy concerns the frequencies relative to the laminarturbulent transition which are shifted to higher frequency with an increase of 1 or $2 \mathrm{kHz}$. Nevertheless the previously described transition phenomenon is similar between these two cases.

The comparison of the normalized PSD function of the spanwise velocity fluctuations relative to the probes located within the wake shows a significant modification of spectra, especially for frequencies lower than $10 \mathrm{kHz}$ (Figure 18a and Figure 18b). Thanks to the incoming turbulence, there are more additional interactions between the incoming, the ejected and the wake vortices. It induces a spectrum with more frequencies, especially around $10 \mathrm{kHz}$ (probe 7). As the coherent structures resulting from the vortex shedding and the laminar separation bubble interact, the freestream turbulence modifies also the spectrum downstream (probes 8 and 9). With freestream turbulence, three main peaks emerge instead of two and the energy of the peaks is differently distributed. 


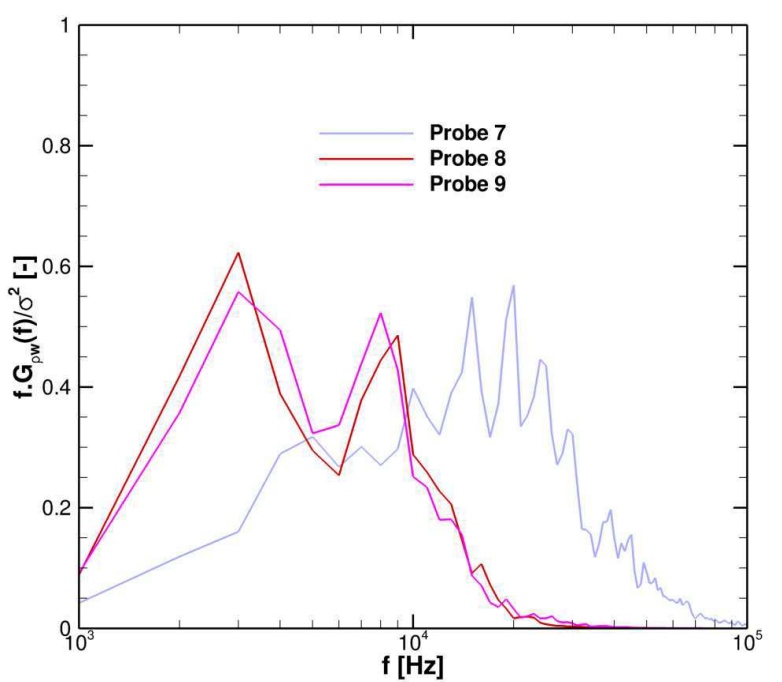

(a) LES w/o freestream turbulence - $\operatorname{Re}_{2 \text { is }}$ $=80000$

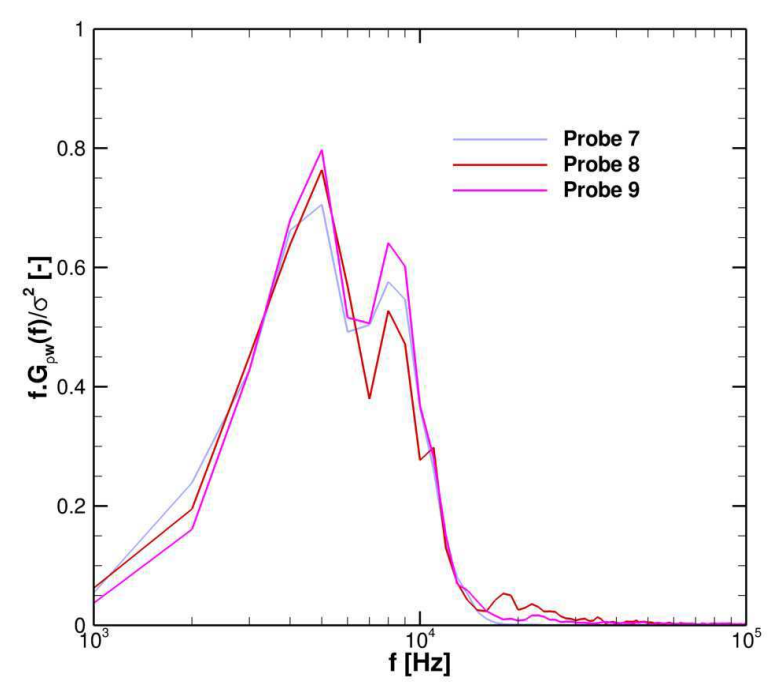

(c) URANS - $\operatorname{Re}_{2 \text { is }}=80000$

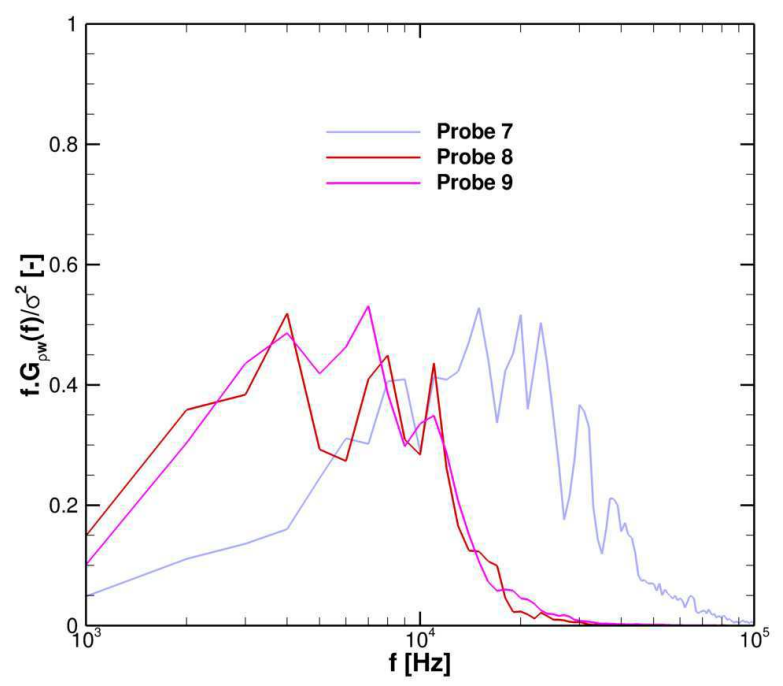

(b) LES w/ freestream turbulence - $\mathrm{Re}_{2 \mathrm{is}}$ $=80000$

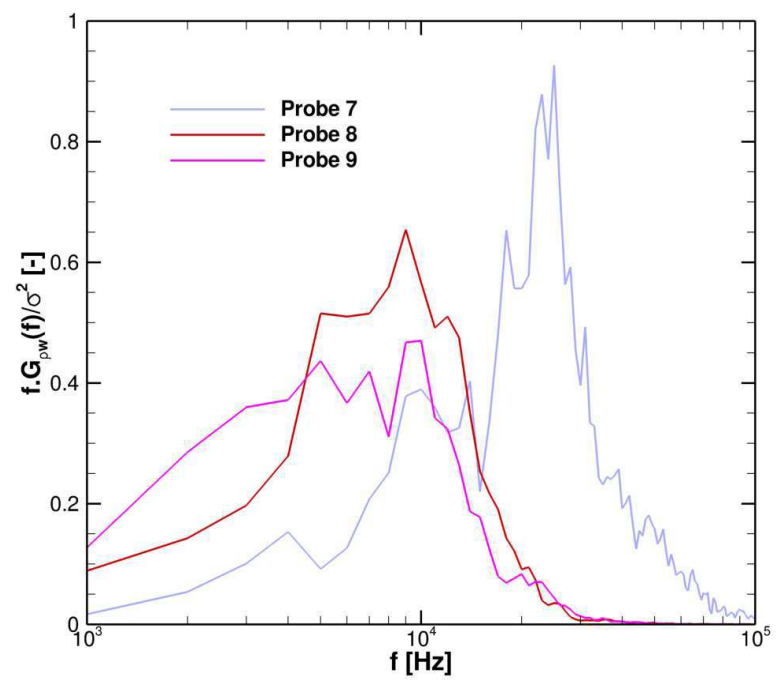

(d) LES w/ freestream turbulence $-\mathrm{Re}_{2 \text { is }}$ $=140000$

Figure 18: Power spectral density functions of spanwise velocity at $\operatorname{Re}_{2 \text { is }}=80000$ and $\operatorname{Re}_{2 \text { is }}=$ 140000 (LES and URANS)

The isentropic exit Reynolds number has a more significant impact than the freestream turbulence (Figure 17b and Figure 17d). Although the laminar separation bubble is still long, this one is thinner for $\operatorname{Re}_{2 \text { is }}=140000$ than for $\operatorname{Re}_{2 \text { is }}=80000$. Thus the transition mechanism induced by the Kelvin-Helmholtz instability and the shear layer flapping involves smaller coherent structures which are ejected from the separation bubble or shed at the trailing edge. It results in higher frequency downstream of the trigger of the transition i.e. downstream of probe 3: the amplitude of frequencies between 16 and $30 \mathrm{kHz}$ is higher. Downstream of the trailing edge (from probe 8), the interaction between the vortices induces a decrease of the amplitude of these frequencies. This interaction is also visible in Figure 18d. The comparison of the normalized PSD function of the spanwise velocity fluctuations shows a significant effect of the Reynolds number on the spectral content of the wake (Figure 18b and Figure $18 d$ ). The coherent structures are smaller for $\operatorname{Re}_{2 i s}=140000$ (Figure 14c) than $\operatorname{Re}_{2 \text { is }}=80000$ (Figure 14b). 
In URANS results (Figure $17 \mathrm{c}$ ), at $\operatorname{Re}_{2 \mathrm{is}}=80000$, the energy relative to the TS instability is distributed over two frequencies due to the resolution frequency: 4 and $5 \mathrm{kHz}$. The $\mathrm{KH}$ frequencies are well captured by URANS. The relative energy contained at these frequencies is even higher than LES although the global energy due to fluctuations in URANS simulation is lower than LES (Figure 15c, also in section 5.2). Nevertheless, downstream, small structures are dissipated. Thus the URANS simulation is unable to capture the breakdown to turbulence as turbulence is not resolved. Only the $5 \mathrm{kHz}$ frequency is observed in the vicinity of the trailing edge and in the wake. This observation is confirmed by the PSD of spanwise velocity fluctuations (Figure 18c). Only two peaks are observed in the wake from the trailing edge. So no vortex pairing occurs in the wake.

As shown by the spanwise velocity fluctuation (Figure 4a), the flow is two-dimensional at the laminar separation point. Thus a criterion of two-dimensional laminar separation can be used. The non-dimensional frequency of the transition mechanism can be represented by a Strouhal number which is defined by:

$$
S t=\frac{f \theta_{S}}{U_{e S}}
$$

Where $\theta$ is the momentum thickness, $U_{e}$ the velocity at the boundary layer edge and the subscript $S$ is relative to the separation point. According to several authors [64][65][56][33], the value of the Strouhal number $S t$ is ranged from 0.005 to 0.008 . In the present study, these values are obtained with the frequencies ranged from 11 to $16 \mathrm{kHz}$ according to the simulated case as shown in Table 4. It confirms that these frequencies are tied to the laminar-turbulent transition due to the Kelvin-Helmholtz instability.

Table 4: Strouhal numbers

\begin{tabular}{|l|c|c|c|}
\hline Isentropic exit Reynolds number & 80000 & 80000 & 140000 \\
\hline Freestream turbulence & No & Yes & Yes \\
\hline Frequency [kHz] & \multicolumn{3}{|c|}{ Strouhal number } \\
\hline 11 & 0.006468 & 0.006556 & 0.00495 \\
\hline 12 & 0.007056 & 0.007152 & - \\
\hline 16 & - & - & 0.0072 \\
\hline
\end{tabular}

Another criteria based on the vorticity thickness $\delta_{\omega}$ (equation (13)) can be applied to validate the frequency of the Kelvin-Helmholtz instability. According to Huerre and Rossi [66], the theoretical Strouhal number $\mathrm{St}_{\delta \omega}$ (equation (14)) relative to Kelvin-Helmholtz instability of classical mixing layer is 0.135 . In the present study, the Strouhal numbers range from 0.11 to 0.12 and are close to the theoretical value. Thus, the instability observed in this investigation is the Kelvin-Helmholtz one.

$$
\begin{aligned}
& \delta_{\omega}=\frac{\Delta U}{\max _{y}\left[\frac{\partial U(x, y, z)}{\partial y}\right]} \quad \text { with } \quad \Delta U=\max _{y}[U(x, y, z)]-\min _{y}[U(x, y, z)] \\
& S t_{\delta_{\omega}}=\frac{f \delta_{\omega}}{\Delta U / 2}
\end{aligned}
$$

The most amplified frequency of TS waves is observed in all simulations, especially at probes located on the wall. The viscous instability is important from the leading to the trailing edge. 
Nevertheless the relative importance of the TS wave in comparison to the inviscid instability changes as we move downstream towards separation. There is a switch-over of the dominant instability, from the TS one (wall mode) to the inviscid one (inflectional mode). In probe 3' located quite far from the wall, these two modes have the same relative energy and the inviscid mode becomes the $\mathrm{KH}$ instability as shown by the criterion computations. Further downstream, at the separated layer edge which is far from the wall, the energy at $\mathrm{KH}$ frequency is higher than the TS one. As shown by Diwan and Ramesh [32], the inflectional instability of the separation bubble originates from the attached boundary layer. The transition process described earlier is linked to the growth of the relative importance of the $\mathrm{KH}$ instability (from probe 3'). Although the TS mode is present, the transition mechanism is mainly driven by the $\mathrm{KH}$ instability, especially the breakdown to turbulence because the mechanism occurs far from the wall.

\section{Conclusion}

This investigation focused on the ability of RANS simulation with transition model and LES method to predict the laminar separation bubble in the rear part of the suction side of the highlift low-pressure turbine blade T106C.

For the lowest studied Reynolds number $\left(\operatorname{Re}_{2 \mathrm{is}}=80000\right)$, all simulations predict a long laminar separation bubble, except the fully turbulent RANS computation. The separation point predicted by LES or RANS simulation with transition model is slightly downstream of the experimental one. Due to the discrepancy in bubble prediction, the kinetic energy loss levels are underestimated by all simulations, especially the RANS simulation on the fine mesh. Nevertheless LES improves the prediction isentropic Mach number distribution and kinetic energy losses at midspan with respect to RANS simulation with transition model of Menter et al. [13]. For LES computations, the freestream turbulence has a small influence on the bubble prediction. This is due to the small intensity of turbulence $(0.9 \%)$. The study of the Reynolds number $\operatorname{Re}_{2 \text { is }}=140000$ shows that the LES simulation overestimates the bursting Reynolds number while the RANS simulation underestimates it. With regard to the numerical cost (CPU hours), it should be noticed that the LES over RANS ratio is about 1000 with the finest mesh. Thus the RANS simulation predicts the laminar separation bubble with a sufficiently accuracy only with the correlation functions calibrated for similar cases and accurate turbulent boundary conditions while LES predicts the laminar separation bubble without transition modeling but a high computational cost.

The unsteady analysis of LES results highlights the role of the separated shear layer on the laminar-turbulent transition within a separation bubble and the large vortices ejected from this bubble. The characteristic frequencies of the involved mechanism are determined thanks to the spectral analysis. The transition mechanism is tied to the Kelvin-Helmholtz instability. Although the Tollmien-Schlichting instability is observed, as the shear layer is far from the wall, the transition mechanism is mainly driven by the Kelvin-Helmholtz instability. This piece of information cannot be obtained with RANS simulation while URANS simulations capture the KH instability but are unable to predict the breakdown to turbulence as turbulence and transition are only modeled with transport equations.

For future work, the transition mechanism will be further investigated focusing on the influence of the mesh density in the area of the separation point and of the mixing layer. Another perspective of this work is to study the influence of incoming wakes, high freestream turbulence and technological effects as film cooling in high-pressure turbine on the transition mechanism and on the boundary layer development. 


\section{Acknowledgements}

This work was granted access to the HPC resources of CCRT under the allocation 2011t2011026662 made by GENCI (Grand Equipement National de Calcul Intensif). The experimental results reported in this study were provided by the Von Karman Institute in the framework of the European research programs TATMo (www.tatmo.eu) and UTAT (Unsteady Transition in Axial Turbines) for which ONERA was also involved. The author is indebted to V. Brunet (ONERA) for his fruitful contribution and to J. Michálek and T. Arts (VKI) for the time trace data. He thanks gratefully F. Richez (ONERA) for the useful discussions concerning this study.

\section{Declaration of conflicting interests}

The author declares that there is no conflict of interest.

\section{Nomenclature}

\section{Latin alphabet}

\begin{tabular}{|c|c|}
\hline$c$ & Blade chord \\
\hline$C_{a x}$ & Axial blade chord \\
\hline$f$ & Frequency \\
\hline$G_{p}(f)$ & Power Spectral Density (PSD) of static pressure \\
\hline$G_{\rho w}(f)$ & PSD of spanwise momentum \\
\hline gradRoSRo & Density gradient normalized by density $\left(\frac{\|\operatorname{grad}(\rho)\|}{\rho}\right.$ \\
\hline$h$ & Characteristic length of shear layer \\
\hline$k$ & Instability wave number \\
\hline$l$ & Turbulent length scale \\
\hline$M$ & Mach number \\
\hline$P$ & Pressure \\
\hline $\operatorname{Re}$ & Reynolds number \\
\hline$S$ & Entropy \\
\hline$S t$ & Strouhal number \\
\hline$T$ & Period \\
\hline Tu & Turbulent rate \\
\hline$U$ & Velocity \\
\hline$v$ & Turbulent velocity scale \\
\hline$x$ & Streamwise distance \\
\hline$y$ & Wall-normal distance \\
\hline$z$ & Spanwise distance \\
\hline \multicolumn{2}{|c|}{ Greek alphabet } \\
\hline$\gamma$ & Specific heat ratio \\
\hline$\delta_{\omega}$ & Vorticity thickness \\
\hline$\Delta$ & Cell volume \\
\hline$\Delta U$ & Velocity variation \\
\hline$\eta$ & Wall distance \\
\hline$\theta$ & Momentum thickness \\
\hline$\lambda$ & Wave length \\
\hline$\lambda_{\theta}$ & Local pressure gradient parameter \\
\hline$\mu_{s g}$ & Subgrid viscosity \\
\hline$\xi$ & Mass-weighted kinetic energy loss coefficient \\
\hline
\end{tabular}




$\begin{array}{ll}\rho & \text { Density } \\ \tau & \text { Turbulent time scale } \\ \text { Subscripts } & \\ 01 & \text { Total quantity in inlet plane } \\ 02 & \text { Total quantity in exit plane } \\ 2 & \text { Exit value } \\ e & \text { Value at boundary layer edge } \\ \text { infl } & \text { Value at inflectional point } \\ \text { max }(k) & \text { Value at turbulent kinetic energy maximum } \\ S & \text { Value at separation point } \\ \text { is } & \text { Isentropic value } \\ \infty & \text { Upstream value } \\ \text { Superscripts } & \\ + & \text { Non-dimensional length in wall units } \\ \text { Acronyms } & \\ C F L & \text { Courant-Friedrichs-Lewy } \\ D N S & \text { Direct Numerical Simulation } \\ K H & \text { Kelvin-Helmholtz instability } \\ L E S & \text { Large-Eddy Simulation } \\ L P T & \text { Low-Pressure Turbine } \\ L S B & \text { Laminar Separation Bubble } \\ P S D & \text { Power Spectral Density } \\ R A N S & \text { Reynolds-Averaged Navier-Stokes } \\ R F G & \text { Random Flow Generation (Smirnov method) } \\ R M S & \text { Root Mean Square } \\ S S T & \text { Shear-Stress Transport (k- } \omega \text { Menter model) } \\ T S & \text { Tollmien-Schlichting instability } \\ W A L E & \text { Wall Adapting Local Eddy viscosity } \\ & \end{array}$

\section{References}

[1] A. Smirnov, S. Shi, and I. Celik. Random flow generation technique for Large Eddy simulations and Particle-Dynamics Modelling. Journal of Fluids Engineering, 123(2):359-371, 2001.

[2] H.P. Hodson and R.J. Howell. Unsteady flow: its role in the low pressure turbine. 9th International Symposium on Unsteady Aerodynamics, Aeroacoustics and Aeroelasticity of Turbomachines, Lyon, France, 2000.

[3] R.E. Mayle. The Role of Laminar-Turbulent Transition in Gas Turbine Engines. Journal of Turbomachinery, 113(4):509-536, October 1991.

[4] M. Gaster. The Structure and Behavior of Laminar Separation Bubbles. ARC R\&M 3595, 1969

[5] A. Hatman and T. Wang. Separated Flow Transition. Part 1. Experimental Methodology and Mode Classification. ASME Turbo Expo 1998, June 2-5, Stockholm, Sweden, ASME Paper 1998-GT-461, 1998.

[6] A. Hatman and T. Wang. Separated Flow Transition. Part2. Experimental Results. ASME Turbo Expo 1998, June 2-5, Stockholm, Sweden, ASME Paper 1998-GT-462, 1998.

[7] A. Hatman and T. Wang. Separated Flow Transition. Part3. Primary Modes and Vortex Dynamics. ASME Turbo Expo 1998, June 2-5, Stockholm, Sweden, ASME Paper 1998-GT-463, 1998.

[8] A. Hatman and T. Wang. A Prediction Model for Separated-Flow Transition. ASME Turbo Expo 1998, June 2-5, Stockholm, Sweden, ASME Paper 1998-GT-237, 1998. 
[9] T. Zaki, P. Durbin, J. Wissink, and W. Rodi. Direct numerical simulation of by-pass and separation induced transition in a linear compressor cascade. ASME Turbo Expo 2006, May 8-11, Barcelona, Spain, May 2006.

[10] J.G. Wissink. DNS of separating, low Reynolds number flow in a turbine cascade with incoming wakes. International Journal of Heat and Fluid Flow, 24(4):626-635, 2003.

[11] J.G. Wissink and W. Rodi. Direct Numerical Simulations of Transitional Flow in Turbomachinery. Journal of Turbomachinery, 128(4):668-678, 2006.

[12] J. Marty, G. Cottin, and B. Aupoix. Steady Numerical Investigations of the Transition Process on an Axial Multistage High Pressure Compressor. ERCOFTAC Bulletin, 80:41-44, September 2009.

[13] F.R. Menter, R.B. Langtry, S.R. Likki, Y.B. Suzen, P.G. Huang, and S. Völker. A Correlation-Based Transition Model Using Local Variables Part I - Model Formulation. Journal of Turbomachinery, 128:413-422, July 2006.

[14] R.B. Langtry, F.R. Menter, S.R. Likki, Y.B. Suzen, P.G. Huang, and S. Völker. A Correlation-Based Transition Model Using Local Variables Part II - Test Cases and Industrial Applications. Journal of Turbomachinery, 128:423-434, July 2006.

[15] A. Benyahia, L. Castillon, and R. Houdeville. Prediction of Separation-Induced Transition on High Lift Low Pressure Turbine Blade. ASME Turbo Expo 2011, June 6-10, Vancouver, Canada, Volume 5: Heat Transfer, Parts A and B of ASME, Paper GT2011-45566, pp. 1835-1846, 2011.

[16] C. Hah. Large Eddy Simulation of Transonic Flow Field in NASA Rotor 37. Technical Memorandum NASA/TM-2009-215627, NASA, 2009.

[17] J. Boudet, J. Caro, and M.C. Jacob. Large-Eddy Simulation of a Low-Speed TipClearance Flow. In M. Sen, G. Bois, M. Manna, and T. Arts, editors, 9th European Conference on Turbomachinery Fluid Dynamics and Thermodynamics, pages 147158, Istanbul, Turkey, 21-25 March, 2011.

[18] J. Riou, A. Benyahia, and V. Brunet. Large Eddy Simulation of Wall Heat Fluxes on a High Pressure Turbine Vane. 46th Symposium of Applied Aerodynamics, Orléans, France, 28-30 March, 2011.

[19] F. Richez, I. Mary, V. Gleize, and C. Basdevant. Zonal RANS/LES coupling simulation of a transitional and separated flow around an airfoil near stall. Theoretical and Computational Fluid Dynamics, 22:305-315, 2008.

[20] B. Raverdy, I. Mary, P. Sagaut, and N. Liamis. High-Resolution Large-Eddy Simulation of Flow Around Low-Pressure Turbine Blade. AIAA Journal, 41(3):390397, 2003.

[21] R. Mittal, S. Venkatasubramanian, and F.M. Najjar. Large-Eddy Simulation of Flow Through a Low-Pressure Turbine Cascade. 15th AIAA CFD Conference, June 11-14, Anaheim, California, Paper AIAA 2001-2560, 2001.

[22] K. Matsuura and C. Kato. Large-Eddy Simulation of compressible transitional cascade flows with and without incoming free-stream turbulence. JSME International Journal Series B Fluids and Thermal Engineering, 49(3):660-669, 2006.

[23] K. Funazaki, K. Yamada, N. Tanaka, and Y. Chiba. Detailed Studies on Separated Boundary layers over Low-Pressure Turbine Airfoils under Several High Lift Conditions: Effect of Freestream Turbulence. ASME Turbo Expo 2009, June 8-12, Orlando, Florida, ASME Paper GT2009-59813, 2009.

[24] W. Bechara, C. Bailly, P. Lafon, and S. Candel. Stochastic approach to noise modeling for free turbulent flows. AIAA Journal, 32(3):455-463, 1994.

[25] V. Michelassi, J.G. Wissink, J. Fröhlich, and W. Rodi. Large-Eddy Simulation of Flow Around Low-Pressure Turbine Blade with Incoming Wakes. AIAA Journal, 41(11):2143-2156, 2003. 
[26] S. Sarkar and P. R. Voke. Large-Eddy Simulation of Unsteady Surface Pressure Over a Low-Pressure Turbine Blade due to Interactions of Passing Wakes and Inflexional Boundary Layer. Journal of Turbomachinery, 128(2):221-231, 2006.

[27] X. F. Zhang and H. Hodson. Effects of Reynolds number and freestream turbulence intensity on the unsteady boundary layer development on an ultra-high-lift low pressure turbine airfoil. Journal of Turbomachinery, 132:011016, January 2010.

[28] C. Fureby, G. Tabor, H. Weller, and A.D. Gosman. A comparative study of sub grid scale models in homogeneous isotropic turbulence. Physics of Fluids, 9(5):1416-1429, 1997.

[29] N. Jarrin, S. Benhamadouche, D. Laurence, and R. Prosser. A synthetic-eddy-method for generating inflow conditions for Large-Eddy Simulations. International Journal of Heat and Fluid Flow, 27(4):585 - 593, 2006.

[30] M. Pamiès, P.E. Weiss, E. Garnier, S. Deck, and P. Sagaut. Generation of synthetic turbulent inflow data for Large Eddy Simulation of spatially evolving wall-bounded flows. Physics of Fluids, 21(4):045103, 2009.

[31] G.R. Tabor and M.H. Baba-Ahmadi. Inlet conditions for Large Eddy Simulation: A review. Computers \& Fluids, 39(4):553 - 567, 2010.

[32] S. S. Diwan and O. N. Ramesh. On the origin of the inflectional instability of a laminar separation bubble. Journal of Fluid Mechanics, 629:263-298, 52009.

[33] B.R. McAuliffe and M.I. Yaras. Numerical Study of Instability Mechanisms Leading to Transition in Separation Bubbles. Journal of Turbomachinery, 130:021006, April 2008.

[34] J. Michálek, M. Monaldi, and T. Arts. Aerodynamic Performance of a Very High Lift Low Pressure Turbine Airfoil (T106C) at Low Reynolds and High Mach Number With Effect of Free Stream Turbulence Intensity. Journal of Turbomachinery, 134:061009, November 2012.

[35] J. Michálek, M. Monaldi, and T. Arts. Aerodynamic Performance of a Very High Lift Low Pressure Turbine Airfoil (T106C) at Low Reynolds and High Mach Number With Effect of Free Stream Turbulence Intensity. ASME Conference Proceedings 2010(44021):1433-1444, 2010, Paper no. GT2010-22884.

[36] L. Cambier, S. Heib, and S. Plot. The Onera elsA CFD software: input from research and feedback from industry. Mechanics \& Industry, eFirst: 1-16, 52013.

[37] I. Mary and P. Sagaut. Large Eddy Simulation of Flow Around an Airfoil Near Stall. AIAA Journal, 40(6):1139-1145, June 2002.

[38] F. Daude. Méthode d'intégration temporelle implicite pour la simulation des grandes échelles application à la réduction du bruit de cavité. $\mathrm{PhD}$ thesis, Université de Poitiers, 2007.

[39] F. Daude, I. Mary, and P. Comte. Local optimization of the convergence rates of implicit time advancements for LES of complex flows. 36th AIAA Fluid Dynamics Conference and Exhibit, 5-8 June 2006, San Francisco, California, AIAA 2006-3545, 2006.

[40] P.L. Roe. Approximate Riemann solver, parameter vector and differences schemes. Journal of Computational Physics, 43:357-372, 1981.

[41] T. Léonard, F. Duchaine, N. Gourdain, and L.Y.M. Gicquel. Steady/Unsteady Reynolds Averaged Navier-Stokes and Large Eddy Simulations of a Turbine Blade at High Subsonic Outlet Mach Number. ASME Conference Proceedings, 2010(44021):697-709, 2010. Paper no. GT2010-22469.

[42] P. Sagaut and S. Deck. Large Eddy Simulation for aerodynamics: status and perspectives. Phil. Trans. R. Soc., 367(1899):2849-2860, 2009.

[43] F.R. Menter. Two-equation eddy-viscosity turbulence models for engineering applications. AIAA Journal, 32(8):1598-1605, August 1994. 
[44] C. Content and R. Houdeville. Local correlation-based transition model. 8th International ERCOFTAC Symposium on Engineering Turbulence Modelling and Measurements, June 9-11 2010.

[45] R.B. Langtry and F.R. Menter. Correlation-Based Transition Modeling for Unstructured Parallelized Computational Fluid Dynamics Codes. AIAA Journal, 47(12):2894-2906, 2009.

[46] F. Ducros, F. Nicoud, and T. Poinsot. Wall-adapting local eddy-viscosity models for simulations in complex geometries. In Baines M. J., editor, ICFD, pages 293-300, Oxford University Computing Lab., UK, 1998.

[47] F. Nicoud and F. Ducros. Subgrid-scale stress modelling based on the square of the velocity gradient tensor. Flow, Turbulence and Combustion, 62(3):183-200, 1999.

[48] V. Brunet. Random Flow Generation Technique for Civil Aircraft Jet Simulations with the ZDES Approach. 4th Symposium on Hybrid RANS-LES Methods, 28-30 September 2011, Beijing, China, 2011.

[49] R.H. Kraichnan. Diffusion by a Random Velocity Field. Physics of Fluids, 13:22-31, 1970.

[50] J. Babajee and T. Arts. Investigation of the laminar separation-induced transition with the $\gamma-R_{\theta T}$ transition model on low-pressure turbine rotor blades at steady conditions. ASME Turbo Expo 2012, June 11-15, Copenhagen, Denmark, Volume 8: Turbomachinery, Parts A, B, and C of ASME, Paper GT2012-68687, pages pp. 11671178, 2012.

[51] V. Marciniak, E. Kuegeler, and M. Franke. Predicting transition on low-pressure turbine profiles. $\mathrm{V}$ European Conference on Computational Fluid Dynamics ECCOMAS CFD, 01622, 2010.

[52] R. Corral and F. Gisbert. Prediction of separation-induced transition using a correlation-based transition model. ASME Turbo Expo 2010: June 14-18, Glasgow, UK, GT2010-23239, 2010.

[53] R. Pacciani, M. Marconcini, A. Arnone, and F. Bertini. Predicting high-lift LP turbine cascades flows using transition-sensitive turbulence closures. ASME Turbo Expo 2013, June 3-7, San Antonio, Texas, USA, GT2013-95605, 2013.

[54] Y. Ooba, H. Kodama, C. Arakawa, Y. Matsuo, and H. Fujiwara. Large-Eddy Simulation of a Low-Pressure Turbine Cascade. 41th Aerospace Science Meeting and Exhibit, AIAA, January 6-9, Reno, Nevada, Paper AIAA 2003-1213, 2003.

[55] B.R. McAuliffe and M.I. Yaras. Transition Mechanisms in Separation Bubbles Under Low- and Elevated-Freestream Turbulence. Journal of Turbomachinery, 132(1):011004-011004-10, September 2009.

[56] J. H. Watmuff. Evolution of a wave packet into vortex loops in a laminar separation bubble. Journal of Fluid Mechanics, 397:119-169, October 1999.

[57] Z. Yang and P. R. Voke. Large-eddy simulation of boundary-layer separation and transition at a change of surface curvature. Journal of Fluid Mechanics, 439:305-333, July 2001.

[58] S. K. Roberts and M. I. Yaras. Large-eddy simulation of transition in a separation bubble. Journal of Fluids Engineering, 128(2):232-238, 2006.

[59] S. Chandrasekhar. Hydrodynamic and Hydromagnetic Stability. Dover Publications, 1981.

[60] P. D. Welch. The Use of Fast Fourier Transform for the Estimation of Power Spectra: a Method Based on Time Averaging over Short, Modified Periodograms. IEEE Transactions on audi and electroacoustics, 2 (AU-15): 70-73, 1967.

[61] W. K. George, P. Beuther and R. E. A. Arndt. Pressure spectra in turbulent free shear flows. Journal of Fluid Mechanics, 148:155-191, 1984, doi: 10.1017/S0022112084002299. 
[62] J. Walker. Transitional flow on axial turbomachine blading. AIAA Journal, 27(5):595602, May 1989.

[63] X. De Saint Victor. Numerical Simulations of Unsteady Separation Bubbles over Turbine Blades. International Journal of Fluid Mechanics Research, 39:40-53, 2012.

[64] L. L. Pauley, P. Moin, and W. C. Reynolds. The structure of two-dimensional separation. Journal of Fluid Mechanics, 220:397-411, 1990.

[65] M. D. Ripley and L. L. Pauley. The unsteady structure of two-dimensional steady laminar separation. Physics of Fluids A: Fluid Dynamics, 5(12):3099-3106, 1993.

[66] P. Huerre and M. Rossi. Hydrodynamic instabilities in open flows. In C. Godrèche and P. Manneville, editors, Hydrodynamics and Nonlinear Instabilities, chapter 2, pages 81-294. Cambridge University Press, 1998. 